\title{
ORBIT EQUIVALENCE RIGIDITY FOR PRODUCT ACTIONS
}

\author{
DANIEL DRIMBE
}

\begin{abstract}
Let $\Gamma_{1}, \ldots, \Gamma_{n}$ be hyperbolic, property (T) groups, for some $n \geq 1$. We prove that if a product $\Gamma_{1} \times \cdots \times \Gamma_{n} \curvearrowright X_{1} \times \cdots \times X_{n}$ of measure preserving actions is stably orbit equivalent to a measure preserving action $\Lambda \curvearrowright Y$, then $\Lambda \curvearrowright Y$ is induced from an action $\Lambda_{0} \curvearrowright Y_{0}$ such that there exists a direct product decomposition $\Lambda_{0}=\Lambda_{1} \times \cdots \times \Lambda_{n}$ into $n$ infinite groups. Moreover, there exists a measure preserving action $\Lambda_{i} \curvearrowright Y_{i}$ that is stably orbit equivalent to $\Gamma_{i} \curvearrowright X_{i}$, for any $1 \leq i \leq n$, and the product action $\Lambda_{1} \times \cdots \times \Lambda_{n} \curvearrowright Y_{1} \times \cdots \times Y_{n}$ is isomorphic to $\Lambda_{0} \curvearrowright Y_{0}$.
\end{abstract}

\section{INTRODUCTION}

An important topic in ergodic theory is the classification of probability measure preserving (pmp) actions up to orbit equivalence. Two pmp actions $\Gamma \curvearrowright(X, \mu)$ and $\Lambda \curvearrowright(Y, \nu)$ are called orbit equivalent $(\mathrm{OE})$ if there exists a measure space isomorphism $\theta:(X, \mu) \rightarrow(Y, \nu)$ which preserves the orbits, i.e. $\theta(\Gamma x)=\Lambda \theta(x)$, for almost every $x \in X$. The classification of actions up to OE is driven by the following fundamental question: what aspects of the group $\Gamma$ and of the action $\Gamma \curvearrowright(X, \mu)$ are remembered by the orbit equivalence relation $\mathcal{R}_{\Gamma \curvearrowright X}:=\{(x, y) \in X \times X \mid \Gamma x=\Gamma y\}$ ?

Equivalence relations $\mathcal{R}_{\Gamma \curvearrowright X}$ tend to forget a lot of information about the groups and actions they are constructed from. This is best illustrated by H. Dye's theorem asserting that any two ergodic pmp actions of $\mathbb{Z}$ are OE Dy58. D.S. Orstein and B. Weiss have extended this result to the class of countable amenable groups [OW80] (see also [CFW81] for a generalization). Consequently, pmp actions of amenable groups manifest a striking lack of rigidity: any algebraic property of the group (e.g. being finitely generated or torsion free) and any dynamical property of the action (e.g. being mixing or weakly mixing) is completely lost in the passage to equivalence relations.

In the non-amenable case, the situation is radically different. More precisely, various properties of the group $\Gamma$ or of the action $\Gamma \curvearrowright(X, \mu)$ can be recovered from the equivalence relation $\mathcal{R}_{\Gamma \curvearrowright X}$. R. Zimmer's pioneering work led to such OE rigidity results for actions of higher rank lattices in semisimple Lie groups. In particular, he showed that if $m, n \geq 3$, then $S L_{m}(\mathbb{Z}) \curvearrowright \mathbb{T}^{m}$ is $\mathrm{OE}$ to $S L_{n}(\mathbb{Z}) \curvearrowright \mathbb{T}^{n}$ if and only if $m=n$ Zi84. Remarkably, by building upon Zimmer's ideas, A. Furman showed that most pmp ergodic actions $\Gamma \curvearrowright(X, \mu)$ of higher rank lattices, including $S L_{n}(\mathbb{Z}) \curvearrowright \mathbb{T}^{n}$, for $n \geq 3$, are OE superrigid [Fu99a, Fu99b]. Roughly speaking, this means that both the group $\Gamma$ and the action $\Gamma \curvearrowright(X, \mu)$ are completely remembered by the equivalence relation $\mathcal{R}_{\Gamma \curvearrowright X}$. By using his influential deformation/rigidity theory, S. Popa showed that any Bernoulli action of a property (T) group [P005] or of a product group [Po06] is OE superrigid. Subsequently, several impressive OE superrigidity results have been discovered in [MS02, Ki06, Io08, IV08, Ki09, PS09, Io14, TD14, CK15, Dr15, GITD16.

However, in general, one can only expect to recover certain aspects of an action $\Gamma \curvearrowright(X, \mu)$ from its orbit equivalence relation $\mathcal{R}_{\Gamma \curvearrowright X}$. For instance, D. Gaboriau showed that the rank of a free group $\mathbb{F}_{n}$ is an invariant of the OE relation $\mathcal{R}_{\mathbb{F}_{n} \curvearrowright X}$ Ga99]. In [MS02], N. Monod and Y. Shalom obtained a series of $\mathrm{OE}$ rigidity results for actions of products $\Gamma=\Gamma_{1} \times \cdots \times \Gamma_{n}$ of hyperbolic groups. In

The author was partially supported by PIMS fellowship. 
particular, they showed that if a product $\Gamma=\Gamma_{1} \times \cdots \times \Gamma_{m}$ of non-elementary hyperbolic torsionfree groups is measure equivalent to a product $\Lambda=\Lambda_{1} \times \cdots \times \Lambda_{n}$ of torsion-free groups (i.e. $\Gamma$ and $\Lambda$ admit stably orbit equivalent actions, see Definition 2.8), then $m \geq n$, and if $m=n$ then, after a permutation of indices, $\Gamma_{i}$ is measure equivalent to $\Lambda_{i}$, for any $1 \leq i \leq n$ (see [MS02, Theorem 1.16] and [Sa09, Theorem 3]). See the surveys [Sh04, Po07, Fu09, Ga10, Va10, Io12, Io17] for an overview on orbit equivalence rigidity results and related topics.

The goal of this paper is to present a new type of rigidity phenomenon in orbit equivalence. Thus, we provide a large class of product actions whose orbit equivalence relation remember the product structure. Before stating the result, let us recall some terminology.

Definition 1.1. Let $\Gamma \curvearrowright(X, \mu)$ and $\Lambda \curvearrowright(Y, \nu)$ be free ergodic pmp actions.

- The actions are stably orbit equivalent (SOE) with index $t$ if there exists a measure space isomorphism $\theta: X_{0} \rightarrow Y_{0}$ between some non-null subsets $X_{0} \subset X$ and $Y_{0} \subset Y$ such that $\theta\left(X_{0} \cap \Gamma x\right)=Y_{0} \cap \Lambda \theta(x)$, for almost every $x \in X_{0}$. Moreover, $t$ is equal to $\nu\left(Y_{0}\right) / \mu\left(X_{0}\right)$.

- We say that $\Lambda \curvearrowright Y$ is induced from an action $\Lambda_{0} \curvearrowright Y_{0}$ of a subgroup $\Lambda_{0}<\Lambda$ if $Y_{0} \subset Y$ is a $\Lambda_{0}$-invariant measurable non-null subset such that $\nu\left(g Y_{0} \cap Y_{0}\right)=0$, for all $g \in \Lambda \backslash \Lambda_{0}$. In this case, note that $\left[\Lambda: \Lambda_{0}\right]=\nu\left(Y_{0}\right)^{-1}$.

Remark 1.2. We will use the following observation about induction. Let $\Lambda \curvearrowright(Y, \nu)$ be a free ergodic pmp action.

- If $\Lambda \curvearrowright Y$ is induced from $\Lambda_{1} \curvearrowright Y_{1}$ and $\Lambda_{1} \curvearrowright Y_{1}$ is induced from $\Lambda_{0} \curvearrowright Y_{0}$, then $\Lambda \curvearrowright Y$ is induced from $\Lambda_{0} \curvearrowright Y_{0}$.

- If $\Lambda \curvearrowright Y$ is induced from $\Lambda_{0} \curvearrowright Y_{0}$, then $\Lambda \curvearrowright Y$ is SOE to $\Lambda_{0} \curvearrowright Y_{0}$ with index $\nu\left(Y_{0}\right)^{-1}$.

Theorem A. Let $\Gamma_{1}, \ldots, \Gamma_{n}$ be property $(T)$, biexact, weakly amenable groups and denote $\Gamma=$ $\Gamma_{1} \times \ldots \times \Gamma_{n}$. For every $1 \leq i \leq n$, let $\Gamma_{i} \curvearrowright\left(X_{i}, \mu_{i}\right)$ be a free ergodic pmp action, and let $\Gamma \curvearrowright(X, \mu)$ be the product action $\Gamma_{1} \times \cdots \times \Gamma_{n} \curvearrowright\left(X_{1} \times \ldots \times X_{n}, \mu_{1} \times \ldots \times \mu_{n}\right)$.

Let $\Lambda \curvearrowright(Y, \nu)$ be a free ergodic pmp action of an icc group which is $S O E$ to $\Gamma \curvearrowright(X, \mu)$ with index $t$, for some $t>0$.

Then $\Lambda \curvearrowright Y$ is induced from an action $\Lambda_{0} \curvearrowright Y_{0}$ and $\Lambda_{0}=\Lambda_{1} \times \ldots \times \Lambda_{n}$ decomposes into a direct product of $n$ infinite groups. Moreover, there exist a pmp action $\Lambda_{i} \curvearrowright\left(Y_{i}, \nu_{i}\right)$ and a positive number $t_{i}$, for any $1 \leq i \leq n$, with $t_{1} \ldots t_{n}=t /\left[\Lambda: \Lambda_{0}\right]$ such that:

(1) $\Lambda_{0} \curvearrowright Y_{0}$ is isomorphic to $\Lambda_{1} \times \ldots \times \Lambda_{n} \curvearrowright Y_{1} \times \ldots \times Y_{n}$.

(2) $\Lambda_{i} \curvearrowright Y_{i}$ is SOE to $\Gamma_{i} \curvearrowright X_{i}$ with index $t_{i}$, for all $1 \leq i \leq n$.

Note that hyperbolic groups are weakly amenable and biexact, see for example the introductions of [PV11, PV12] for an extensive discussion on these notions. Remark that the following classes of groups are property $(\mathrm{T})$, biexact, weakly amenable groups:

- uniform lattices in $S p(m, 1)$ with $m \geq 2$ or any group in their measure equivalence class,

- Gromov's random groups with density satisfying $3^{-1}<d<2^{-1}$ [Gr93, Zu03].

A result due to Singer [Si55] provides an approach to the study of orbit equivalence of actions using von Neumann algebras. In this context, we use a combination of techniques from S. Popa's deformation/rigidity theory to prove Theorem A.

Our main result can be seen as the orbit equivalence analog of a striking theorem of I. Chifan, R. de Santiago and T. Sinclair [CdSS15, Theorem A] in which they show that the group von Neumann algebra $L(\Gamma)$ of a product of non-elementary hyperbolic groups completely remembers the product structure of the generating group $\Gamma$. 
Theorem A complements the following remarkable result of N. Monod and Y. Shalom MS02, Theorem 1.9]. First, we recall that a pmp action $\Lambda \curvearrowright(Y, \nu)$ is mildly mixing if there are no nontrivial recurrent subsets: if a measurable subset $A \subset Y$ satisfies $\liminf _{g \rightarrow \infty} \nu(g A \Delta A)=0$, then $\nu(A) \in\{0,1\}$.

Theorem 1.3 ([MS02]). Let $\Gamma=\Gamma_{1} \times \Gamma_{2}$ be a product of torsion-free non-elementary hyperbolic groups and let $\Gamma \curvearrowright X$ be a free irreducible pmp action. Assume $\Gamma \curvearrowright X$ is orbit equivalent to a mildly mixing action $\Lambda \curvearrowright Y$, where $\Lambda$ is a torsion-free group.

Then there exists a group isomorphism $\delta: \Gamma \rightarrow \Lambda$ and a measure space isomorpshism $\theta: X \rightarrow Y$ such that $\theta(g x)=\delta(g) \theta(x)$, for all $g \in \Gamma$ and almost every $x \in X$.

Remark that Monod and Shalom's result applies to the class of irreducible actions $\Gamma_{1} \times \Gamma_{2} \stackrel{\sigma}{\curvearrowright} X$, i.e. $\sigma_{\mid \Gamma_{i}}$ is ergodic for any $1 \leq i \leq 2$, while Theorem $\mathrm{A}$ provides orbit equivalence rigidity results for product actions.

Comments on the proof of Theorem $\mathbf{A}$. We outline briefly and informally the proof of our main result. Assume for simplicity that $\Gamma=\Gamma_{1} \times \Gamma_{2}$ is a product of two hyperbolic, property (T) groups. Let $\Gamma_{i} \curvearrowright X_{i}$ be a free ergodic pmp action, for any $1 \leq i \leq 2$. Denote by $\Gamma \curvearrowright X$ the product action $\Gamma_{1} \times \Gamma_{2} \curvearrowright X_{1} \times X_{2}$ and assume that it is OE to a free aperiodic action $\Lambda \curvearrowright Y$, where $\Lambda$ is an icc group. By a result of Singer [Si55] (see also [FM75]) there exists an isomorphism of the groups measure space von Neumann algebras, $L^{\infty}(X) \rtimes \Gamma \cong L^{\infty}(Y) \rtimes \Lambda$, which identifies $L^{\infty}(X)$ and $L^{\infty}(Y)$. Hence, we assume $M:=L^{\infty}(X) \rtimes \Gamma=L^{\infty}(Y) \rtimes \Lambda$ and $L^{\infty}(X)=L^{\infty}(Y)$.

Following [PV09, we define the comultiplication $*$-homomorphism $\Delta: M \rightarrow M \bar{\otimes} L(\Lambda)$ by letting $\Delta\left(a v_{g}\right)=a v_{g} \otimes v_{g}$, for all $a \in L^{\infty}(X), g \in \Lambda$. We use the relative strong solidity property of hyperbolic groups (see Section 2.5) obtained in the breakthrough work of S. Popa and S. Vaes [PV11, PV12] and the property (T) assumption of $\Gamma_{1}$ to deduce that there exists $j \in\{1,2\}$ such that

$$
\Delta\left(L\left(\Gamma_{1}\right)\right) \prec_{M \bar{\otimes} M} M \bar{\otimes}\left(L^{\infty}\left(X_{j}\right) \rtimes \Gamma_{j}\right) .
$$

Here, $P \prec{ }_{N} Q$ denotes that a corner of $P$ embeds into $Q$ inside $N$, in the sense of Popa [Po03].

A key step of the proof consists in applying A. Ioana's ultrapower technique from [o11] (see Theorem 4.2) which allows us to deduce from (1.1) the existence of a subgroup $\Sigma<\Lambda$ such that

$$
L^{\infty}(X) \rtimes \Gamma_{1} \prec_{M} L^{\infty}(X) \rtimes \Sigma \text { and the centralizer of } \Sigma \text { in } \Lambda \text { is non-amenable. }
$$

By using (1.2) together with the relative strong solidity of the groups $\Gamma_{i}$ 's, we obtain that there exists a non-zero projection $e \in\left(L^{\infty}(X) \rtimes \Sigma\right)^{\prime} \cap M$ such that

$$
L^{\infty}(X) \rtimes \Gamma_{1} \prec_{M}\left(L^{\infty}(X) \rtimes \Sigma\right) f \text { and }\left(L^{\infty}(X) \rtimes \Sigma\right) f \prec_{M} L^{\infty}(X) \rtimes \Gamma_{1},
$$

for all $f \in\left(L^{\infty}(X) \rtimes \Sigma\right)^{\prime} \cap M$ with $f \leq e$.

Next, by applying [DHI16, Proposition 3.1] to (1.3) we derive that $\Sigma$ is measure equivalent to $\Gamma_{1}$, and hence, obtain that $\Sigma$ has property (T). Inspired by techniques from [CdSS15] we further deduce that, up to replacing $\Sigma$ by a finite index subgroup, the subgroup generated by $\Sigma$ and its centralizer $C_{\Lambda}(\Sigma)$ has finite index in $\Lambda$. Finally, by using once again (1.3) and adapting some arguments from [CdSS15], we conclude that $\Lambda \curvearrowright Y$ is induced from an action $\Lambda_{0} \curvearrowright Y_{0}$ that admits a direct product decomposition $\Lambda_{1} \times \Lambda_{2} \curvearrowright Y_{1} \times Y_{2}$ such that $\Lambda_{i} \curvearrowright Y_{i}$ is stably orbit equivalent to $\Gamma_{i} \curvearrowright X_{i}$, for any $1 \leq i \leq 2$.

Acknowledgment. I would like to thank Adrian Ioana for many comments that helped improve the exposition of the paper. I am also grateful to the referees for carefully reading the paper, for all their suggestions and for pointing out an error in an earlier version of Theorem 3.1. 


\section{Preliminaries}

2.1. Terminology. In this paper we consider tracial von Neumann algebras $(M, \tau)$, i.e. von Neumann algebras $M$ equipped with a faithful normal tracial state $\tau: M \rightarrow \mathbb{C}$. This induces a norm on $M$ by the formula $\|x\|_{2}=\tau\left(x^{*} x\right)^{1 / 2}$, for all $x \in M$. We will always assume that $M$ is a separable von Neumann algebra, i.e. the $\|\cdot\|_{2}$-completion of $M$ denoted by $L^{2}(M)$ is separable as a Hilbert space. We denote by $\mathcal{U}(M)$ the unitary group of $M$ and by $\mathcal{Z}(M)$ its center.

All inclusions $P \subset M$ of von Neumann algebras are assumed unital. We denote by $E_{P}: M \rightarrow P$ the unique $\tau$-preserving conditional expectation from $M$ onto $P$, by $P^{\prime} \cap M=\{x \in M \mid x y=$ $y x$, for all $y \in P\}$ the relative commutant of $P$ in $M$ and by $\mathcal{N}_{M}(P)=\left\{u \in \mathcal{U}(M) \mid u P u^{*}=P\right\}$ the normalizer of $P$ in $M$. We say that $P$ is regular in $M$ if the von Neumann algebra generated by $\mathcal{N}_{M}(P)$ equals $M$. For two von Neumann subalgebras $P, Q \subset M$, we denote by $P \vee Q$ the von Neumann algebra generated by $P$ and $Q$.

The amplification of a $\mathrm{II}_{1}$ factor $(M, \tau)$ by a positive number $t$ is defined to be $M^{t}=p\left(\mathbb{B}\left(\ell^{2}(\mathbb{Z})\right) \bar{\otimes} M\right) p$, for a projection $p \in \mathbb{B}\left(\ell^{2}(\mathbb{Z})\right) \bar{\otimes} M$ satisfying $(\operatorname{Tr} \otimes \tau)(p)=t$. Here $\operatorname{Tr}$ denotes the usual trace on $\mathbb{B}\left(\ell^{2}(\mathbb{Z})\right)$. Since $M$ is a $\mathrm{II}_{1}$ factor, $M^{t}$ is well defined. Note that if $M=P_{1} \bar{\otimes} P_{2}$, for some $\mathrm{II}_{1}$ factors $P_{1}$ and $P_{2}$, then there exists a natural identification $M=P_{1}^{t} \bar{\otimes} P_{2}^{1 / t}$, for every $t>0$.

Let $\Gamma \stackrel{\sigma}{\curvearrowright} A$ be a trace preserving action of a countable group $\Gamma$ on a tracial von Neumann algebra $(A, \tau)$. For a subgroup $\Sigma<\Gamma$, we denote by $A^{\Sigma}=\left\{a \in A \mid \sigma_{g}(a)=a\right.$, for all $\left.g \in \Sigma\right\}$, the subalgebra of elements of $A$ fixed by $\Sigma$.

For a countable group $\Gamma$ and for two subsets $S, T \subset \Gamma$, we denote by $\langle S\rangle$ the group generated by $S$, and by $C_{S}(T)=\{g \in S \mid g h=h g$, for all $h \in T\}$ the centralizer of $T$ in $S$.

For an abelian von Neumann algebra $A=L^{\infty}(X)$ and a measurable subset $X_{0} \subset X$, we denote by $1_{X_{0}}$ the associated projection in $A$.

2.2. Intertwining-by-bimodules. We next recall from [Po03, Theorem 2.1 and Corollary 2.3] the powerful intertwining-by-bimodules technique of S. Popa.

Theorem 2.1 ([Po03]). Let $(M, \tau)$ be a tracial von Neumann algebra and $P \subset p M p, Q \subset q M q$ be von Neumann subalgebras. Let $\mathcal{U} \subset \mathcal{U}(P)$ be a subgroup such that $\mathcal{U}^{\prime \prime}=P$.

Then the following are equivalent:

(1) There exist projections $p_{0} \in P, q_{0} \in Q, a *$-homomorphism $\theta: p_{0} P p_{0} \rightarrow q_{0} Q q_{0}$ and a non-zero partial isometry $v \in q_{0} M p_{0}$ such that $\theta(x) v=v x$, for all $x \in p_{0} P p_{0}$.

(2) There is no sequence $\left(u_{n}\right)_{n} \subset \mathcal{U}$ satisfying $\left\|E_{Q}\left(x u_{n} y\right)\right\|_{2} \rightarrow 0$, for all $x, y \in M$.

Notation 2.2. Throughout the paper we will use the following notation.

- If one of the equivalent conditions of Theorem 2.1 holds true, we write $P \prec_{M} Q$, and say that a corner of $P$ embeds into $Q$ inside $M$.

- If $P p^{\prime} \prec_{M} Q$ for any non-zero projection $p^{\prime} \in P^{\prime} \cap p M p$ (equivalently, for any non-zero projection $p^{\prime} \in \mathcal{N}_{p M p}(P)^{\prime} \cap p M p$, by Lemma 2.5(1)), then we write $P \prec_{M}^{s} Q$.

- If $P \prec_{M} Q q^{\prime}$ for any non-zero projection $q^{\prime} \in Q^{\prime} \cap q M q$ (equivalently, for any non-zero projection $q^{\prime} \in \mathcal{N}_{q M q}(Q)^{\prime} \cap q M q$, by Lemma 2.5(2)), then we write $P \prec_{M}^{s^{\prime}} Q$.

We continue with some results containing several elementary facts regarding Popa's intertwiningby-bimodules technique. 
Lemma 2.3. Let $(M, \tau)$ be a tracial von Neumann algebra and let $N \subset M$ be a von Neumann subalgebra. Let $P \subset p N p$ and $Q \subset q N q$ be von Neumann subalgebras such that $P \prec_{N}^{s^{\prime}} Q$.

Then $P \prec_{M}^{s^{\prime}} Q$.

Note that Lemma 2.3 also holds true if we replace the symbol $\prec^{s^{\prime}}$ by $\prec^{s}$ as shown in DHI16, Remark $2.2]$.

Proof. Let $q^{\prime} \in Q^{\prime} \cap q M q$ be a non-zero projection. Let $q^{\prime \prime} \in Q^{\prime} \cap q N q$ be the support projection of $E_{N}\left(q^{\prime}\right)$. The assumption implies that $P \prec_{N} Q q^{\prime \prime}$, hence there exist projections $p_{0} \in P, q_{0} \in Q$, a $*$-homomorphism $\theta: p_{0} P p_{0} \rightarrow q_{0} Q q_{0} q^{\prime \prime}$ and a non-zero partial isometry $v \in q_{0} q^{\prime \prime} N p_{0}$ such that $\theta(x) v=v x$, for all $x \in p_{0} P p_{0}$. Since $q^{\prime} \leq q^{\prime \prime}$, we let $\tilde{\theta}: p_{0} P p_{0} \rightarrow q_{0} Q q_{0} q^{\prime}$ be the $*$-homomorphism defined by $\tilde{\theta}(x)=\theta(x) q^{\prime}$, for all $x \in p_{0} P p_{0}$. By denoting $\tilde{v}=q^{\prime} v$, we have $\tilde{\theta}(x) \tilde{v}=\tilde{v} x$, for all $x \in p_{0} P p_{0}$. Note that $\tilde{v}$ is non-zero since $E_{N}\left(q^{\prime}\right) v \neq 0$. Finally, by replacing $\tilde{v}$ by the partial isometry from its polar decomposition, we obtain that $P \prec_{M} Q q^{\prime}$.

Lemma 2.4. Let $(M, \tau)$ be a tracial von Neumann algebra and let $P \subset p M p, Q \subset q M q, R \subset r M r$ be von Neumann subalgebras. Then the following hold:

(1) Assume that $P \prec_{M} Q$ and $Q \prec_{M}^{s} R$. Then $P \prec_{M} R$, Va08, Lemma 3.7].

(2) Assume $P \prec_{M}^{s^{\prime}} Q$ and $Q \prec_{M} R$. Then $P \prec_{M} R$.

Proof. We will prove only the second statement.

(2) The assumption $Q \prec_{M} R$ implies that there exist projections $q_{0} \in Q, r_{0} \in R$, a non-zero partial isometry $v \in r_{0} M q_{0}$ and a $*$-homomorphism $\psi: q_{0} Q q_{0} \rightarrow r_{0} R r_{0}$ such that $\psi(x) v=v x$, for all $x \in q_{0} Q q_{0}$. Let $q^{\prime}$ be the support projection of $v^{*} v$ and note that $q^{\prime} \in Q^{\prime} \cap q M q$ and $q^{\prime} \leq q_{0}$. Without loss of generality, we can assume that the support projection of $E_{R}\left(v v^{*}\right)$ equals $r_{0}$. The assumption implies that $P \prec_{M} Q q^{\prime}$, hence there exist projections $p_{0} \in P, q_{1} \in Q$, a non-zero partial isometry $w \in q_{1} q^{\prime} M p_{0}$ and a $*$-homomorphism $\phi: p_{0} P p_{0} \rightarrow q_{1} Q q_{1} q^{\prime}$ such that $\phi(x) w=w x$, for all $x \in p_{0} P p_{0}$. We can assume that the support projection of $E_{Q q^{\prime}}\left(w w^{*}\right)$ equals $q^{\prime} q_{1}$.

Since $q_{0} Q q_{0} q^{\prime}=Q q^{\prime}$, we can define the $*$-homomorphism $\psi^{\prime}: Q q^{\prime} \rightarrow r_{0} R r_{0}$ by letting $\psi^{\prime}\left(x q^{\prime}\right)=$ $\psi(x)$, for all $x \in q_{0} Q q_{0}$. Notice that $\psi^{\prime}$ is well defined because if $x q^{\prime}=0$, for some $x \in q_{0} Q q_{0}$, then $\psi(x) v q^{\prime}=0$. This shows that $\psi(x) E_{R}\left(v v^{*}\right)=0$, which implies $\psi(x)=0$. Moreover, it is clear that $\psi^{\prime}\left(x q^{\prime}\right) v q^{\prime}=v q^{\prime}\left(x q^{\prime}\right)$, for all $x \in q_{0} Q q_{0}$. We continue by noticing that $v q^{\prime} w \neq 0$. Indeed, by assuming the contrary, we obtain that $q^{\prime} E_{Q q^{\prime}}\left(w w^{*}\right)=0$, which implies that $q^{\prime} q_{1}=0$, contradiction.

Finally, remark that the $*$-homomorphism $\psi^{\prime} \circ \phi: p_{0} P p_{0} \rightarrow r_{0} R r_{0}$ satisfies $\left(\psi^{\prime} \circ \phi\right)(x) v q^{\prime} w=v q^{\prime} w x$, for all $x \in p_{0} P p_{0}$. By replacing $v q^{\prime} w$ by the partial isometry from its polar decomposition, we deduce that $P \prec_{M} R$.

We will use repeatedly the following results from [DHI16] and Va08] and we record them in the following combined lemma for reader's convenience.

Lemma 2.5. Let $(M, \tau)$ be a tracial von Neumann algebra and let $P \subset p M p, Q \subset q M q$ be von Neumann subalgebras. Then the following hold:

(1) If $P z \prec_{M} Q$, for any non-zero projection $z \in \mathcal{N}_{p M p}(P)^{\prime} \cap p M p$, then $P \prec_{M}^{s} Q$, [DHI16, Lemma 2.4(2)].

(2) If $P \prec_{M} Q$, then there exists a non-zero projection $z \in \mathcal{N}_{q M q}(Q)^{\prime} \cap q M q$ such that $P \prec_{M}^{s^{\prime}} Q z$, DHI16, Lemma 2.4(4)].

(3) If $P \prec_{M} Q$, then $Q^{\prime} \cap q M q \prec_{M} P^{\prime} \cap p M p$, [Va08, Lemma 3.5]. 
If we consider two commuting subalgebras $P_{1}$ and $P_{2}$ of $M$ that both embed into a subalgebra $Q$ of $M$ in the sense of Popa (see Theorem 2.1), we would like to obtain that $P_{1} \vee P_{2}$ embeds into $Q$. In general this is not true. For instance, consider the crossed product $M=(P \bar{\otimes} P) \rtimes_{\sigma} \mathbb{Z} / 2 \mathbb{Z}$ with the period two automorphism $\sigma(a \otimes b)=b \otimes a$, where $P$ is a tracial diffuse von Neumann algebra. Then, we have that $P \otimes 1 \prec_{M} P \otimes 1$ and $1 \otimes P \prec_{M} P \otimes 1$, but $P \bar{\otimes} P \nprec_{M} P \otimes 1$. The following lemma essentially shows that in the presence of a weak regularity condition on $Q$, the result is true. Although its proof is easy, this lemma plays an important role in the paper.

Lemma 2.6. Let $M$ be a von Neumann algebra and let $P_{1}, P_{2} \subset M$ and $Q \subset q M q$ be von Neumans subalgebras such that $\mathcal{N}_{q M q}(Q)^{\prime} \cap q M q=\mathbb{C} q$. Suppose there exist commuting subalgebras $\tilde{P}_{0}, \tilde{P}_{1}, \tilde{P}_{2} \subset M$ such that $P_{1} \subset \tilde{P}_{1}, P_{2} \subset \tilde{P}_{2}$ and $\tilde{P}_{0} \vee \tilde{P}_{1} \vee \tilde{P}_{2}=M$.

If $P_{i} \prec_{M} Q$, for any $i \in\{1,2\}$, then $P_{1} \vee P_{2} \prec_{M} Q$.

Proof. The assumption implies that there exist projections $p \in P_{1}, q_{0} \in Q$, a $*$-homomorphism $\varphi: p P_{1} p \rightarrow q_{0} Q q_{0}$ and a non-zero partial isometry $v \in q_{0} M p$ such that $\varphi(x) v=v x$, for every $x \in p P_{1} p$.

We aim to show that $\left(p P_{1} p\right) \vee\left(P_{2} p\right) \prec_{M} Q$. By supposing the contrary, there exist two sequences of unitaries $\left(u_{n}\right)_{n} \subset \mathcal{U}\left(p P_{1} p\right)$ and $\left(v_{n}\right)_{n} \subset \mathcal{U}\left(P_{2}\right)$, such that

$$
\left\|E_{Q}\left(x u_{n}\left(v_{n} p\right) y\right)\right\|_{2} \rightarrow 0, \text { for all } x, y \in M .
$$

Since $M=\tilde{P}_{0} \vee \tilde{P}_{1} \vee \tilde{P}_{2}$, we obtain that

$$
\left\|E_{Q}\left(x u_{n} y v_{n} z\right)\right\|_{2} \rightarrow 0, \text { for all } x, y, z \in M .
$$

Using that $\varphi\left(u_{n}\right) v=v u_{n}$, for any $n \geq 1$, we have $\| E_{Q}\left(v u_{n} y v_{n} z\left\|_{2}=\right\| E_{Q}\left(v y v_{n} z\right) \|_{2}\right.$, for all $y, z \in M$. Combining this last remark with relation (2.1), we obtain that $\left\|E_{Q}\left(a y v_{n} z\right)\right\|_{2} \rightarrow 0$, for all $y, z \in M$, where $a:=v v^{*} \in q M q$. Since $w E_{Q}(x) w^{*}=E_{Q}\left(w x w^{*}\right)$, for any $x \in M$ and $w \in \mathcal{N}_{q M q}(Q)$, it follows that

$$
\left\|E_{Q}\left(\left(w a w^{*}\right) y v_{n} z\right)\right\|_{2} \rightarrow 0, \text { for all } y, z \in M \text { and } w \in \mathcal{N}_{q M q}(Q)
$$

We continue by noticing that for any two projections $p_{1}$ and $p_{2}$ in $M$, we have $p_{1} \vee p_{2}=s\left(p_{1}+p_{2}\right)$. Here we denote by $s(b)$ the support projection of an an element $b \in M$. Moreover, by using Borel functional calculus, there exist a sequence $\left(c_{n}\right)_{n} \subset M$ such that $\left(p_{1}+p_{2}\right) c_{n}$ converges to $s\left(p_{1}+p_{2}\right)$ in the $\|\cdot\|_{2}$-norm. Thus, it follows by (2.2) that $\left\|E_{Q}\left(\left(w_{1} a w_{1}^{*}+w_{2} a w_{2}^{*}\right) y v_{n} z\right)\right\|_{2} \rightarrow 0$, and hence, $\left\|E_{Q}\left(\left(w_{1} a w_{1}^{*} \vee w_{2} a w_{2}^{*}\right) y v_{n} z\right)\right\|_{2} \rightarrow 0$, for any $w_{1}, w_{2} \in \mathcal{N}_{q M q}(Q)$ and $y, z \in M$.

Therefore, we obtain by induction that

$$
\left\|E_{Q}\left(\left(w_{1} a w_{1}^{*} \vee \ldots \vee w_{n} a w_{n}^{*}\right) y v_{n} z\right)\right\|_{2} \rightarrow 0,
$$

for any $w_{1}, \ldots, w_{n} \in \mathcal{N}_{q M q}(Q)$ and $y, z \in M$.

Finally, remark that $\vee_{w \in \mathcal{N}_{q M q}(Q)} w a w^{*}=q$ since $\mathcal{N}_{q M q}(Q)^{\prime} \cap q M q=\mathbb{C} q$. Hence, it follows that $\left\|E_{Q}\left(y v_{n} z\right)\right\|_{2} \rightarrow 0$, for all $y, z \in M$. This shows that $P_{2} \nprec_{M} Q$, contradiction.

The next lemma is well known. We include only a sketch of the proof since the result follows, for example, from [HPV11, Lemma 1] (see also [Po01, Theorem 6.2]).

Lemma 2.7. Let $M$ and $M_{0}$ be some tracial von Neumann algebras and let $A \subset M$ be an abelian subalgebra. Denote $\mathcal{M}=M_{0} \bar{\otimes} M$.

If $P \subset p \mathcal{M} p$ is a property (T) subalgebra such that $P \prec_{\mathcal{M}} M_{0} \bar{\otimes} A$, then $P \prec_{\mathcal{M}} M_{0}$. 
Proof. Since $A$ is abelian, take an increasing sequence of finite dimensional abelian algebras $A_{n}$, $n \geq 1$. Using the fact that $P$ has property (T) and $P \prec_{\mathcal{M}}\left(\cup_{n} M_{0} \bar{\otimes} A_{n}\right)^{\prime \prime}$, we get that there exists an integer $n_{0}$ such that $P \prec \mathcal{M} M_{0} \bar{\otimes} A_{n_{0}}$. This proves the claim since $A_{n_{0}}$ is finite dimensional.

Property $(\mathrm{T})$ for von Neumann algebras was defined by Connes and Jones in [CJ85. Note that a countable group $\Gamma$ has property $(\mathrm{T})$ if and only if $L(\Gamma)$ has property $(\mathrm{T})$ CJ85, Po01.

The following result is a tool that was discovered in [DHI16, Proposition 3.1] which allows us to derive from some intertwining relations that certain groups are measure equivalent in the sense of M. Gromov. We first recall the notion of measure equivalence Gr91, Fu99a].

Definition 2.8. Two countable groups $\Gamma$ and $\Lambda$ are called measure equivalent if there exist free ergodic pmp actions $\Gamma \curvearrowright(X, \mu)$ and $\Lambda \curvearrowright(Y, \nu)$ which are stably orbit equivalent.

Proposition 2.9 ( DHI16]). Let $M$ be a II factor and let $\Gamma \curvearrowright(X, \mu)$ and $\Lambda \curvearrowright(Y, \nu)$ be free ergodic pmp actions such that $p M p=L^{\infty}(X) \rtimes \Gamma$ and $q M q=L^{\infty}(Y) \rtimes \Lambda$, for some projections $p, q \in M$. Suppose that $\Gamma=\Gamma_{1} \times \Gamma_{2}$ and $L^{\infty}(X) \prec_{M} L^{\infty}(Y)$. Assume that there exists a subgroup $\Sigma<\Lambda$ such that the following hold:

- $L^{\infty}(X) \rtimes \Gamma_{1} \prec_{M} L^{\infty}(Y) \rtimes \Sigma$, and

- $L^{\infty}(Y) \rtimes \Sigma \prec_{M}^{s} L^{\infty}(X) \rtimes \Gamma_{1}$.

Then $\Sigma$ is measure equivalent to $\Gamma_{1}$.

2.3. Finite index inclusions of von Neumann algebras. The Jones index for an inclusion $P \subset M$ of $\mathrm{II}_{1}$ factors is the dimension of $L^{2}(M)$ as a left $P$-module [Jo81. M. Pimsner and S. Popa defined a probabilistic notion of index for an inclusion $P \subset M$ of arbitrary von Neumann algebras with conditional expectation, which in the case of inclusions of $\mathrm{II}_{1}$ factors coincides with Jones' index [PP86, Theorem 2.2]. Namely, the inclusion $P \subset M$ of tracial von Neumann algebras is said to have probabilistic index $[M: P]=\lambda^{-1}$, where

$$
\lambda=\inf \left\{\left\|E_{P}(x)\right\|_{2}^{2}\|x\|_{2}^{-2} \mid x \in M_{+}, x \neq 0\right\} .
$$

Here we use the convention that $\frac{1}{0}=\infty$.

We continue with recording several basic facts concerning finite index inclusions of von Neumann algebras. For a proof of the next lemma, see [CIK13, Lemma 2.4], for example.

Lemma 2.10 ([PP86, Lemma 2.3]). Let $N \subset M$ be tracial von Neumann algebras satisfying $[M: N]<\infty$. Then the following hold:

(1) for every projection $p \in N$, we have $[p M p: p N p]<\infty$.

(2) $M \prec_{M}^{s} N$.

Lemma 2.11. Let $N \subset M$ be tracial von Neumann algebras satisfying $[M: N]<\infty$ and assume that $\mathcal{Z}(N)$ is completely atomic.

Then $q M q \prec_{q M q} N q$, for every projection $q \in N^{\prime} \cap M$. Moreover, $q M q \prec_{q M q}^{s^{\prime}} N q$, for every projection $q \in N^{\prime} \cap M$.

Proof. Let $q \in N^{\prime} \cap M$ be a non-zero projection. Let $z \in \mathcal{Z}(N)$ be a projection such that $N z$ is a factor and $q z \neq 0$. Lemma 2.10 (1) implies that $[z M z: N z]<\infty$. Since $N z$ is a

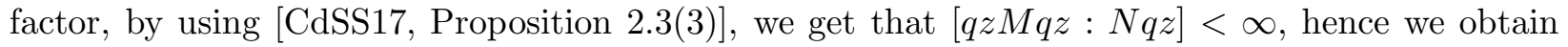
$q z M q z \prec_{q z M q z} N q z$ by Lemma 2.10(2). Therefore, $q M q \prec_{q M q} N q$. Note that the moreover part follows from the first part.

The following lemma goes back to [Jo81] and extends the results from [Jo81, Examples 2.3.2 and 2.3.3]. 
Lemma 2.12. Let $\Gamma \stackrel{\sigma}{\curvearrowright}(A, \tau)$ be a trace preserving action and denote $M=A \rtimes \Gamma$. Let $\left(\Delta_{1}, \Delta_{2}\right)$ and $\left(\Omega_{1}, \Omega_{2}\right)$ be two pairs of commuting subgroups of $\Gamma$ such that $\Delta_{1} \subset \Omega_{1}$ and $\Omega_{2} \subset \Delta_{2}$ are finite index inclusions.

Then the inclusion $A^{\Omega_{1}} \rtimes \Omega_{2} \subset A^{\Delta_{1}} \rtimes \Delta_{2}$ has finite index.

Proof. Note that it is enough to show the following two statements:

(1) $A^{\Omega_{1}} \rtimes \Omega_{2} \subset A^{\Delta_{1}} \rtimes \Omega_{2}$ has finite index.

(2) $A^{\Delta_{1}} \rtimes \Omega_{2} \subset A^{\Delta_{1}} \rtimes \Delta_{2}$ has finite index.

(1) Denote by $\left\{u_{g}\right\}_{g \in \Gamma}$ the canonical unitaries which implement the action $\Gamma \curvearrowright A$. Let $g_{1}, \ldots, g_{n} \in$ $\Omega_{1}$ such that we have the partition $\Omega_{1}=\underset{1 \leq i \leq n}{\sqcup} g_{i} \Delta_{1}$. One can check that the map $E_{0}: A^{\Delta_{1}} \rightarrow A^{\Omega_{1}}$ defined by $E_{0}(a)=\frac{1}{n} \sum_{i=1}^{n} \sigma_{g_{i}}(a)$ is the unique conditional expectation from $A^{\Delta_{1}}$ to $A^{\Omega_{1}}$. Denote by $E: A^{\Delta_{1}} \rtimes \Omega_{2} \rightarrow A^{\Omega_{1}} \rtimes \Omega_{2}$ the conditional expectation from $A^{\Delta_{1}} \rtimes \Omega_{2}$ to $A^{\Omega_{1}} \rtimes \Omega_{2}$. Since $\Omega_{1}$ and $\Omega_{2}$ commute, note that for any $x=\sum_{g \in \Omega_{2}} x_{g} u_{g} \in A^{\Delta_{1}} \rtimes \Omega_{2}$, we have $E(x)=\sum_{g \in \Omega_{2}} E_{0}\left(x_{g}\right) u_{g}=$ $\frac{1}{n} \sum_{i=1}^{n} u_{g_{i}} x u_{g_{i}}^{*}$. Hence, for any $x \in\left(A^{\Delta_{1}} \rtimes \Omega_{2}\right)_{+}$, we have $\|E(x)\|_{2}^{2} \geq \frac{1}{n^{2}} \sum_{i=1}^{n} \tau\left(u_{g_{i}} x^{*} u_{g_{i}}^{*} u_{g_{i}} x u_{g_{i}}^{*}\right)=$ $\frac{1}{n}\|x\|_{2}^{2}$. This ends the first part.

(2) Denote $P=A^{\Delta_{1}} \rtimes \Omega_{2}$ and $N=A^{\Delta_{1}} \rtimes \Delta_{2}$. Denote by $e_{P}: L^{2}(N) \rightarrow L^{2}(P)$ the orthogonal projection onto $L^{2}(P)$ and note that the basic construction $\left\langle N, e_{P}\right\rangle$ is isomorphic to $\left(A^{\Delta_{1}} \bar{\otimes} \ell^{\infty}\left(\Delta_{2} / \Omega_{2}\right)\right) \rtimes \Delta_{2}$ (see, for example, Be14, Lemma 2.5]). Hence, $\left\langle N, e_{P}\right\rangle$ is tracial, since $\left[\Delta_{2}: \Omega_{2}\right]<\infty$. Therefore, there exists a normal conditional expectation $E:\left\langle N, e_{P}\right\rangle \rightarrow N$, which implies that $P \subset N$ is a finite index inclusion.

Lemma 2.13. Let $\Gamma \stackrel{\sigma}{\curvearrowright} A$ be a trace preserving ergodic action on an abelian von Neumann algebra $(A, \tau)$ and let $\Sigma<\Gamma$ be a finite index subgroup.

Then, $A^{\Sigma}$ is completely atomic.

Proof. Let $n=[\Gamma: \Sigma]$ and take $g_{1}, \ldots, g_{n} \in \Gamma$ such that we have the partition $\Gamma=\sqcup_{1 \leq i \leq n} g_{i} \Sigma$. Assume by contrary that $A^{\Sigma}$ is not completely atomic. Then there exists a non-zero projection $p \in A^{\Sigma}$ such that $\tau(p) \leq 1 /(2 n)$. Note that $a:=\sum_{i=1}^{n} \sigma_{g_{i}}(p)$ belongs to $A^{\Gamma}=\mathbb{C}$ and $\tau(a) \leq 1 / 2$. This leads to a contradiction.

2.4. Relative amenability of subalgebras. A tracial von Neumann algebra $(M, \tau)$ is amenable if there exists a positive linear functional $\Phi: \mathbb{B}\left(L^{2}(M)\right) \rightarrow \mathbb{C}$ such that $\Phi_{\mid M}=\tau$ and $\Phi$ is $M$-central, meaning $\Phi(x T)=\Phi(T x)$, for all $x \in M$ and $T \in \mathbb{B}\left(L^{2}(M)\right)$.

A very useful relative version of this notion has been introduced by N. Ozawa and S. Popa in [OP07. Let $(M, \tau)$ be a tracial von Neumann algebra. Let $p \in M$ be a projection and $P \subset p M p, Q \subset M$ be von Neumann subalgebras. Following OP07, Definition 2.2], we say that $P \subset p M p$ is amenable relative to $Q$ inside $M$ if there exists a positive linear functional $\Phi: p\left\langle M, e_{Q}\right\rangle p \rightarrow \mathbb{C}$ such that $\Phi_{\mid p M p}=\tau$ and $\Phi$ is $P$-central. Note that $P$ is amenable relative to $\mathbb{C}$ inside $M$ if and only if $P$ is amenable.

We next record the following useful results:

Lemma 2.14 ([Be14, BV12] $)$. Let $\Gamma \stackrel{\sigma}{\curvearrowright}(X, \mu)$ be a pmp action and denote $A=L^{\infty}(X)$ and $M=L^{\infty}(X) \rtimes \Gamma$. Let $p \in A$ be non-zero projection and $\Sigma<\Gamma$ a subgroup. Let $\mathcal{G}$ be a subgroup of $\mathcal{N}_{p M p}(A p)$.

(1) If $\mathcal{G}^{\prime \prime} \prec_{M} A \rtimes \Sigma$, then $(A p \cup \mathcal{G})^{\prime \prime} \prec_{M} A \rtimes \Sigma$, [BV12, Lemma 2.3]. 
(2) Assume that $\sigma$ is free and $(\mathcal{G} q)^{\prime \prime}$ is amenable relative to $A \rtimes \Sigma$, for some non-zero projection $q \in \mathcal{G}^{\prime} \cap p M p$. Then, $(A p \cup \mathcal{G})^{\prime \prime} q_{0}$ is amenable relative to $A \rtimes \Sigma$, for some non-zero projection $q_{0} \in(A p \cup \mathcal{G})^{\prime} \cap p M p$, Be14, Lemma 2.11].

Proposition 2.15 ([PV11,DHI16]). Let $(M, \tau)$ be a tracial von Neumann algebra and $Q_{1}, Q_{2} \subset M$ von Neumann subalgebras which form a commuting square, i.e. $E_{Q_{1}} \circ E_{Q_{2}}=E_{Q_{2}} \circ E_{Q_{1}}$. Assume that $Q_{1}$ is regular in $M$. Let $P \subset p M p$ be a von Neumann subalgebra. Then the following hold:

(1) If $P \prec_{M}^{s} Q_{1}$ and $P \prec_{M}^{s} Q_{2}$, then $P \prec_{M}^{s} Q_{1} \cap Q_{2}$, [DHI16, Lemma 2.8(2)].

(2) If $P$ is amenable relative to $Q_{1}$ and $Q_{2}$, then $P$ is amenable relative to $Q_{1} \cap Q_{2}$, [PV11, Proposition 2.7].

2.5. Relatively strongly solid groups. Following [CIK13, Definition 2.7], a countable group $\Gamma$ is said to be relatively strongly solid and write $\Gamma \in \mathcal{C}_{r s s}$ if for any trace preserving action $\Gamma \curvearrowright B$ the following holds: if $M=B \rtimes \Gamma$ and $A \subset p M p$ is a von Neumann subalgebra which is amenable relative to $B$ inside $M$, then either $A \prec_{M} B$ or the normalizer $\mathcal{N}_{p M p}(A)^{\prime \prime}$ is amenable relative to $B$ inside $M$.

In their breakthrough work PV11, PV12, S. Popa and S. Vaes proved that non-elementary hyperbolic groups belong to $\mathcal{C}_{\text {rss }}$. More generally, [PV12, Theorem 1.4] shows that all weakly amenable, biexact groups are relatively strongly solid.

The following consequence of belonging to $\mathcal{C}_{\text {rss }}$ will be useful (see [KV15, Lemma 5.2]).

Lemma 2.16 ([KV15]). Let $\Gamma \curvearrowright Q$ be a trace preserving action of a group $\Gamma$ that belongs to the class $\mathcal{C}_{r s s}$, and let $M=Q \rtimes \Gamma$. Let $P_{1}, P_{2} \subset p M p$ be commuting von Neumann subalgebras.

Then either $P_{1} \prec_{M} Q$ or $P_{2}$ is amenable relative to $Q$.

\section{From tensor DeCompositions to DeCompositions of ACtions}

For proving Theorem $\mathrm{A}$ we need the following result, which provides sufficient conditions at the von Neumann algebra level for a pmp action to admit a non-trivial direct product decomposition (see also [Dr19, Theorem 3.1]). The factor setting is essential for the result and its proof is based on arguments from [CdSS15, Theorem 4.14] (see also [DHI16, Theorem 6.1] and [CdSS17, Theorem 4.7]).

Theorem 3.1. Let $\Lambda \curvearrowright(Y, \nu)$ be a free ergodic pmp action of an icc group $\Lambda$. Let $M=L^{\infty}(Y) \rtimes \Lambda$ and assume that $M=P_{1} \bar{\otimes} P_{2}$ for some $I I_{1}$ factors $P_{1}$ and $P_{2}$.

Suppose that there exist infinite commuting subgroups $\Sigma_{1}, \Sigma_{2}<\Lambda$ with $\left[\Lambda: \Sigma_{1} \Sigma_{2}\right]<\infty$ such that

$$
L\left(\Sigma_{1}\right) e \prec_{M} P_{1} \text { and } L\left(\Sigma_{2}\right) e \prec_{M}^{s} P_{2},
$$

for some non-zero projection $e \in L^{\infty}(Y)^{\Sigma_{1} \Sigma_{2}}$.

Then there exist infinite commuting subgroups $\Lambda_{1}, \Lambda_{2}<\Lambda$ such that $\Lambda \curvearrowright Y$ is induced from an action $\Lambda_{1} \Lambda_{2} \curvearrowright Y_{0}$.

Moreover, there exist a decomposition $1_{Y_{0}} M 1_{Y_{0}}=P_{1}^{t_{1}} \bar{\otimes} P_{2}^{t_{2}}$, for some $t_{1}, t_{2}>0$ with $\nu\left(Y_{0}\right)=t_{1} t_{2}$ and a unitary $u \in \mathcal{U}\left(1_{Y_{0}} M 1_{Y_{0}}\right)$ such that

$$
P_{1}^{t_{1}}=u\left(L^{\infty}(Y)^{\Lambda_{2}} \rtimes \Lambda_{1}\right) 1_{Y_{0}} u^{*} \text { and } P_{2}^{t_{2}}=u\left(L^{\infty}(Y)^{\Lambda_{1}} \rtimes \Lambda_{2}\right) 1_{Y_{0}} u^{*} .
$$

In particular, there exists a pmp action $\Lambda_{i} \curvearrowright\left(Y_{i}, \nu_{i}\right)$, for any $i \in\{1,2\}$, such that $\Lambda_{1} \Lambda_{2} \curvearrowright Y_{0}$ is isomorphic to the product action $\Lambda_{1} \times \Lambda_{2} \curvearrowright Y_{1} \times Y_{2}$. 
Before proceeding with the proof, we introduce some terminology and recall a well known lemma. Let $\Sigma<\Lambda$ be a subgroup. Following [CdSS15, we denote by $\mathcal{O}_{\Sigma}(g)=\left\{h g h^{-1} \mid h \in \Sigma\right\}$ the orbit of $g \in \Lambda$ under the conjugation action of $\Sigma$. Note that $\mathcal{O}_{\Sigma}\left(g_{1} g_{2}\right) \subset \mathcal{O}_{\Sigma}\left(g_{1}\right) \mathcal{O}_{\Sigma}\left(g_{2}\right)$, thus $\left|\mathcal{O}_{\Sigma}\left(g_{1} g_{2}\right)\right| \leq\left|\mathcal{O}_{\Sigma}\left(g_{1}\right)\right|\left|\mathcal{O}_{\Sigma}\left(g_{2}\right)\right|$. This implies that the set $\Delta=\left\{g \in \Lambda \mid \mathcal{O}_{\Sigma}(g)\right.$ is finite $\}$ is a subgroup of $\Lambda$. Note also that $\Delta$ is normalized by $\Sigma$. Moreover, one can check that if $\Lambda \curvearrowright(Y, \nu)$ is a pmp action, then $L(\Sigma)^{\prime} \cap\left(L^{\infty}(Y) \rtimes \Lambda\right) \subset L^{\infty}(Y) \rtimes \Delta$.

Lemma 3.2. Let $(M, \tau)$ be a tracial von Neumann algebra and assume $M=P_{1} \bar{\otimes} P_{2}$ for some von Neumann algebras $P_{1}$ and $P_{2}$. Let $A \subset P_{1}$ be a von Neumann subalgebra such that $A \prec_{M} P_{2}$.

Then, $A$ is not diffuse.

Proof. Assume by contradiction that $A$ is diffuse. Therefore, there exists a sequence $\left(u_{n}\right)_{n} \subset A$ of unitaries such that $\tau\left(u_{n} x\right) \rightarrow 0$ for all $x \in A$. This implies that

$$
\left\|E_{P_{2}}\left(x u_{n} y\right)\right\|_{2} \rightarrow 0, \text { for all } x, y \in M .
$$

Indeed, note that since $M=P_{1} \bar{\otimes} P_{2}$, we can assume $x=1$ and $y=y_{1} \otimes y_{2}$ with $y_{1} \in P_{1}$ and $y_{2} \in P_{2}$. Hence, $\left\|E_{P_{2}}\left(x u_{n} y\right)\right\|_{2}=\tau\left(u_{n} y_{1}\right)\left\|y_{2}\right\|_{2} \rightarrow 0$. Therefore, (3.1) is true, which implies the contradiction $A \nprec_{M} P_{2}$.

Proof of Theorem 3.1. Let $A=L^{\infty}(Y)$ and denote $\Delta_{2}=\left\{g \in \Lambda \mid \mathcal{O}_{\Sigma_{1}}(g)\right.$ is finite $\}$. Since $\Sigma_{2} \subset \Delta_{2}$ and $\left[\Lambda: \Sigma_{1} \Sigma_{2}\right]<\infty$, we obtain that there exist $g_{1}, \ldots, g_{n} \in \Delta_{2}$ such that $\Delta_{2} \Sigma_{1}=\cup_{i=1}^{n} g_{i} \Sigma_{2} \Sigma_{1}$. Since $\left[\Sigma_{1}: C_{\Sigma_{1}}\left(g_{i}\right)\right]<\infty$ for every $1 \leq i \leq n$, we obtain that $\Delta_{1}:=\cap_{i=1}^{n} C_{\Sigma_{1}}\left(g_{i}\right)$ is a finite index subgroup of $\Sigma_{1}$. Note that $\Sigma_{1}$ is icc, since $\Lambda$ is icc and $\left[\Lambda: \Sigma_{1} \Sigma_{2}\right]<\infty$. Therefore, $\left[\Delta_{2}: \Sigma_{2}\right]<\infty$ and $\Delta_{1}$ and $\Delta_{2}$ are commuting subgroups of $\Lambda$.

Remark that since $\left[\Sigma_{1}: \Delta_{1}\right]<\infty$, we obtain that $\mathcal{O}_{\Sigma_{1}}(g)$ is finite if and only if $\mathcal{O}_{\Delta_{1}}(g)$ is finite, for any $g \in \Lambda$. This implies that $\left\{g \in \Lambda \mid \mathcal{O}_{\Delta_{1}}(g)\right.$ is finite $\}$ equals $\Delta_{2}$. Hence,

$$
L\left(\Delta_{1}\right)^{\prime} \cap M=\left(A^{\Delta_{2}} \rtimes \Delta_{1}\right)^{\prime} \cap M=A^{\Delta_{1}} \rtimes \Delta_{2} .
$$

Indeed, first note that $L\left(\Delta_{1}\right)^{\prime} \cap M \supset\left(A^{\Delta_{2}} \rtimes \Delta_{1}\right)^{\prime} \cap M \supset A^{\Delta_{1}} \rtimes \Delta_{2}$. Now, take $x \in L\left(\Delta_{1}\right)^{\prime} \cap M$. Since $\left\{g \in \Lambda \mid \mathcal{O}_{\Delta_{1}}(g)\right.$ is finite $\}$ equals $\Delta_{2}$, it follows that $x \in A \rtimes \Delta_{2}$. Using the fact that $\Delta_{1}$ and $\Delta_{2}$ commute, we obtain that $x \in A^{\Delta_{1}} \rtimes \Delta_{2}$, which proves (3.2).

We continue by showing the following claim.

Claim. We have $\left(A^{\Delta_{2}} \rtimes \Delta_{1}\right) e \prec_{M} P_{1}$ and $e\left(A^{\Delta_{1}} \rtimes \Delta_{2}\right) e \prec_{M}^{s} P_{2}$.

Proof of the Claim. The assumption implies that $L\left(\Delta_{1}\right) e \prec_{M} P_{1}$. By passing to relative commutants and by applying twice Lemma 2.5)(3), we obtain that $\left(\left(L\left(\Delta_{1}\right)^{\prime} \cap M\right)^{\prime} \cap M\right) e \prec_{M} P_{1}$. Relation (3.2) readily implies that $\left(A^{\Delta_{2}} \rtimes \Delta_{1}\right) e \prec_{M} P_{1}$.

For proving the second statement, we first show $\left(A^{\Delta_{1}} \rtimes \Sigma_{2}\right) e \prec_{M}^{s} P_{2}$. Denote $\Omega_{1}=\left\{g \in \Lambda \mid \mathcal{O}_{\Sigma_{2}}(g)\right.$ is finite $\}$ and let $\Omega_{2}=C_{\Sigma_{2}}\left(\Omega_{1}\right)$. One can show as before that the inclusions of subgroups $\Delta_{1} \subset$ $\Sigma_{1} \subset \Omega_{1}$ and $\Omega_{2} \subset \Sigma_{2} \subset \Delta_{2}$ are of finite index. As in (3.2), we obtain that $L\left(\Omega_{2}\right)^{\prime} \cap M=A^{\Omega_{2}} \rtimes \Omega_{1}$. Let $f \in \mathcal{N}_{e M e}\left(\left(A^{\Delta_{1}} \rtimes \Sigma_{2}\right) e\right)^{\prime} \cap e M e \subset\left(L\left(\Delta_{1} \Sigma_{2}\right) e\right)^{\prime} \cap e M e=A^{\Delta_{1} \Sigma_{2}}$ e. The last equality follows from the fact that $\left[\Lambda: \Delta_{1} \Sigma_{1}\right]<\infty$ and $\Lambda$ is icc. The assumption implies $L\left(\Omega_{2}\right) f \prec_{M} P_{2}$. As in the previous paragraph, by applying Lemma 2.5)(3), we get that $\left(L\left(\left(\Omega_{2}\right)^{\prime} \cap M\right)^{\prime} \cap M\right) f \prec_{M} P_{2}$. This shows that $\left(A^{\Omega_{1}} \rtimes \Omega_{2}\right) f \prec_{M} P_{2}$, since $L\left(\Omega_{2}\right)^{\prime} \cap M=A^{\Omega_{2}} \rtimes \Omega_{1}$.

Since $\left[\Omega_{1}: \Delta_{1}\right]<\infty$ and $\left[\Sigma_{2}: \Omega_{2}\right]<\infty$, we can use Lemma 2.12 and obtain that $A^{\Omega_{1}} \rtimes \Omega_{2} \subset$ $A^{\Delta_{1}} \rtimes \Sigma_{2}$ has finite index. Note that $\Omega_{1}$ and $\Omega_{2}$ are icc since $\Lambda$ is icc and $\left[\Lambda: \Omega_{1} \Omega_{2}\right]<\infty$. This shows that $\mathcal{Z}\left(A^{\Omega_{1}} \rtimes \Omega_{2}\right)=A^{\Omega_{1} \Omega_{2}}$ and is completely atomic by Corollary 2.13. Hence, Lemma 2.11 combined with Lemma 2.3 imply that $\left(A^{\Delta_{1}} \rtimes \Sigma_{2}\right) f \prec_{M}^{s^{\prime}}\left(A^{\Omega_{1}} \rtimes \Omega_{2}\right) f$. In combination with the 
conclusion of the previous paragraph and by applying Lemma 2.4 we get that $\left(A^{\Delta_{1}} \rtimes \Sigma_{2}\right) f \prec_{M} P_{2}$. This shows that $\left(A^{\Delta_{1}} \rtimes \Sigma_{2}\right) e \prec_{M}^{s} P_{2}$.

Finally, for finishing the proof of the claim, note that Lemma 2.12 shows that $\left[A^{\Delta_{1}} \rtimes \Delta_{2}: A^{\Delta_{1}} \rtimes\right.$ $\left.\Sigma_{2}\right]<\infty$. This gives by applying Lemma 2.10 that $e\left(A^{\Delta_{1}} \rtimes \Delta_{2}\right) e \prec_{M}^{s}\left(A^{\Delta_{1}} \rtimes \Sigma_{2}\right) e$ since $e \in A^{\Delta_{1}}$. Therefore, by applying Lemma 2.4(1) and using the conclusion of the previous paragraph, we obtain that $e\left(A^{\Delta_{1}} \rtimes \Delta_{2}\right) e \prec_{M}^{s} P_{2}$.

Lemma 2.13 shows that $A^{\Delta_{1} \Delta_{2}}$ is completely atomic. Thus, there exists a projection $f \in A^{\Delta_{1} \Delta_{2}}$ such that $A^{\Delta_{1} \Delta_{2}} f=\mathbb{C} f$, ef $\neq 0$ and $\left(A^{\Delta_{2}} \rtimes \Delta_{1}\right)$ ef $\prec_{M} P_{1}$.

Denote $t=\tau(e f)$ and $M_{0}=e f M e f$. Note that we have the identification $M_{0}=M^{t}=P_{1}^{t} \bar{\otimes} P_{2}$. For ease of notation, we denote $Q_{1}=P_{1}^{t}$ and $Q_{2}=P_{2}$.

Note that $\Delta_{1}$ and $\Delta_{2}$ are icc as well and that $\left(A^{\Delta_{2}} \rtimes \Delta_{1}\right)$ ef and $e\left(A^{\Delta_{1}} \rtimes \Delta_{2}\right) e f$ are $\mathrm{II}_{1}$ factors. Thus, by combining the Claim with (3.2), we have that the von Neumann algebras $\left(A^{\Delta_{2}} \rtimes \Delta_{1}\right) e f \prec_{M} Q_{1}$, and $\left(A^{\Delta_{2}} \rtimes \Delta_{1}\right)$ ef and $\left[\left(A^{\Delta_{2}} \rtimes \Delta_{1}\right) e f\right]^{\prime} \cap M_{0}$ are $\mathrm{II}_{1}$ factors. Therefore, we can apply OP03, Proposition 12] and deduce that there exist a decomposition $M_{0}=Q_{1}^{s} \bar{\otimes} Q_{2}^{1 / s}$, for some $s>0$ and a unitary $v \in M_{0}$ such that

$$
v\left(A^{\Delta_{2}} \rtimes \Delta_{1}\right) e f v^{*} \subset Q_{1}^{s} .
$$

Using once again relation (3.2) we obtain that

$$
Q_{2}^{1 / s} \subset v e\left(A^{\Delta_{1}} \rtimes \Delta_{2}\right) e f v^{*} .
$$

By applying [Ge95, Theorem A], we can find a factor $C \subset Q_{1}^{s}$ such that $C \bar{\otimes} Q_{2}^{1 / s}=v e\left(A^{\Delta_{1}} \rtimes\right.$ $\left.\Delta_{2}\right) e f v^{*}$. Note that the Claim shows that $e\left(A^{\Delta_{1}} \rtimes \Delta_{2}\right) e f \prec_{M_{0}} Q_{2}$, which implies that $C \prec_{M_{0}} Q_{2}$. Finally, using that $C \subset Q_{1}^{s}$, we obtain that $C$ is not diffuse by Lemma 3.2. Since $C$ is a factor, it must be finite dimensional, hence $C=\mathbb{M}_{k}(\mathbb{C})$, for some $k \geq 1$. Denoting $t_{0}=s / k$, we get that

$$
Q_{2}^{1 / t_{0}}=v e\left(A^{\Delta_{1}} \rtimes \Delta_{2}\right) e f v^{*} .
$$

Denote $\Omega_{1}=\left\{g \in \Lambda \mid \mathcal{O}_{\Sigma_{2}}(g)\right.$ is finite $\}$ and $\Omega_{2}=C_{\Sigma_{2}}\left(\Omega_{1}\right)$, as in the proof of the Claim. Remark that $\Omega_{1}=\left\{g \in \Lambda \mid \mathcal{O}_{\Delta_{2}}(g)\right.$ is finite $\}$ is normalized by $\Delta_{2}$ and $\Delta_{2}=\left\{g \in \Lambda \mid \mathcal{O}_{\Omega_{1}}(g)\right.$ is finite $\}$ is normalized by $\Omega_{1}$, since $\left[\Delta_{2}: \Sigma_{2}\right]<\infty$ and $\left[\Omega_{1}: \Sigma_{1}\right]<\infty$. Note also that $\Omega_{1} \cap \Delta_{2}=1$ since $\Delta_{2}$ is icc. Hence, any commutator $[g, h]$, with $g \in \Omega_{1}, h \in \Delta_{2}$ belongs to $\Omega_{1} \cap \Delta_{2}=1$. This shows that $\Omega_{1}$ and $\Delta_{2}$ are commuting subgroups.

Using (3.4), we get that $Q_{1}^{t_{0}}=v\left(\left(A^{\Delta_{1}} \rtimes \Delta_{2}\right)^{\prime} \cap M\right) e f v^{*} \subset v e f\left(A^{\Omega_{2}} \rtimes \Omega_{1}\right) e f v^{*}$. It follows that $M_{0}=Q_{1}^{t_{0}} \bar{\otimes} Q_{2}^{1 / t_{0}} \subset \operatorname{vef}\left(A \rtimes \Omega_{1} \Delta_{2}\right) e f v^{*}$. We claim that $\Lambda_{1}:=\Omega_{1}$ and $\Lambda_{2}:=\Delta_{2}$ satisfy the conclusions of the theorem.

Note that the previous inclusion implies that efMef $=$ ef $\left(A \rtimes \Lambda_{1} \Lambda_{2}\right)$ ef. Let $z$ be the central support of ef in $A \rtimes \Lambda_{1} \Lambda_{2}$ and note that $z M z=\left(A \rtimes \Lambda_{1} \Lambda_{2}\right) z$. Denote by $Y_{0} \subset Y$ the $\Lambda_{1} \Lambda_{2^{-}}$ invariant measurable subset such that $z=1_{Y_{0}} \in \mathcal{Z}\left(A \rtimes \Lambda_{1} \Lambda_{2}\right)=A^{\Lambda_{1} \Lambda_{2}}$ and note that $\Lambda \curvearrowright Y$ is induced from $\Lambda_{1} \Lambda_{2} \curvearrowright Y_{0}$, since $z u_{g} z=0$ for any $g \in \Lambda \backslash \Lambda_{1} \Lambda_{2}$. Here, we denote by $\left\{u_{g}\right\}_{g \in \Lambda}$ the canonical unitaries which implement the action $\Lambda \curvearrowright A$.

To this end, we use the following identification $z M z=M^{\tau(z)}=P_{1}^{\tau(z)} \bar{\otimes} P_{2}$. Observe that (3.4) shows that $L\left(\Lambda_{2}\right) z \prec_{M^{\tau(z)}} P_{2}$. By passing to relative commutants and by applying twice Lemma 2.5(3) we obtain that $\left(A^{\Lambda_{1}} \rtimes \Lambda_{2}\right) z \prec_{M^{\tau(z)}} P_{2}$. Therefore, by applying [OP03, Proposition 12] (note that $\left(A^{\Lambda_{1}} \rtimes \Lambda_{2}\right) z$ and $\left(\left(A^{\Lambda_{1}} \rtimes \Lambda_{2}\right) z\right)^{\prime} \cap z M z$ are $\mathrm{II}_{1}$ factors) and [Ge95, Theorem $\mathrm{A}$ ] as before, there exist a decomposition $M^{\tau(z)}=P_{1}^{t_{1}} \bar{\otimes} P_{2}^{t_{2}}$, for some $t_{1}, t_{2}>0$ with $t_{1} t_{2}=\tau(z)$, a unitary $w \in M^{\tau(z)}$, and a von Neumann subalgebra $D \subset P_{2}^{t_{2}}$ such that $P_{1}^{t_{1}} \bar{\otimes} D=w\left(A^{\Lambda_{2}} \rtimes \Lambda_{1}\right) z w^{*}$. Remark that 
Lemma 2.12 and Lemma 2.10 show that $\left[\left(A^{\Lambda_{2}} \rtimes \Lambda_{1}\right) z:\left(A^{\Delta_{2}} \rtimes \Delta_{1}\right) z\right]<\infty$, and Lemma 2.11 implies

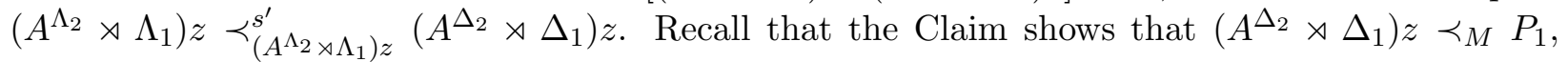
since $e f \leq z$. Now, Lemma 2.3 and Lemma 2.4(2) imply that $\left(A^{\Lambda_{2}} \rtimes \Lambda_{1}\right) z \prec_{M^{\tau(z)}} P_{1}^{t_{1}}$, hence, $D \prec_{M^{\tau(z)}} P_{1}^{t_{1}}$. Since $D \subset P_{2}^{t_{2}}$, it cannot be diffuse by Lemma 3.2. Using that $D$ is factor, it follows that it must be finite dimensional. Therefore, $D=\mathbb{M}_{p}(\mathbb{C})$, for some $p \geq 1$. Thus, $P_{1}^{t_{1} p}=$ $w\left(A^{\Lambda_{2}} \rtimes \Lambda_{1}\right) z w^{*}$. By passing to relative commutants, we obtain that $P_{2}^{t_{2} / p}=w\left(A^{\Lambda_{1}} \rtimes \Lambda_{2}\right) z w^{*}$. This clearly implies the conclusion of the theorem by noticing that $z M z=L^{\infty}\left(Y_{0}\right) \rtimes \Lambda_{1} \Lambda_{2}$ and by representing $A^{\Lambda_{2}} z=L^{\infty}\left(Y_{1}\right)$ and $A^{\Lambda_{1}} z=L^{\infty}\left(Y_{2}\right)$ for some standard probability spaces $\left(Y_{1}, \nu_{1}\right)$ and $\left(Y_{2}, \nu_{2}\right)$.

\section{Proof of Theorem A}

In this section we will prove the von Neumann algebraic version of Theorem $\mathrm{A}$ and use it to derive the main result of the introduction. First, we make the remark that if $\Lambda \curvearrowright Y$ is induced from an action $\Lambda_{0} \curvearrowright Y_{0}$, then we have the identification $L^{\infty}\left(Y_{0}\right) \rtimes \Lambda_{0}^{\left[\Lambda: \Lambda_{0}\right]}=L^{\infty}(Y) \rtimes \Lambda$ since $L^{\infty}\left(Y_{0}\right) \rtimes \Lambda_{0}=1_{Y_{0}}\left(L^{\infty}(Y) \rtimes \Lambda\right) 1_{Y_{0}}$.

Theorem 4.1. Let $\Gamma_{1}, \ldots, \Gamma_{n}$ be property $(T)$, biexact, weakly amenable groups and denote $\Gamma=$ $\Gamma_{1} \times \ldots \times \Gamma_{n}$. For every $1 \leq i \leq n$, let $\Gamma_{i} \curvearrowright\left(X_{i}, \mu_{i}\right)$ be a free ergodic pmp action and denote $M_{i}=L^{\infty}\left(X_{i}\right) \rtimes \Gamma_{i}$ and $M=M_{1} \bar{\otimes} \ldots \bar{\otimes} M_{n}$.

Let $\Lambda \curvearrowright(Y, \nu)$ be a free ergodic pmp action of a countable icc group $\Lambda$ such that $M^{t}=L^{\infty}(Y) \rtimes \Lambda$, for some $t>0$.

Then $\Lambda \curvearrowright Y$ is induced from an action $\Lambda_{0} \curvearrowright Y_{0}$ and there exist a decomposition $\Lambda_{0}=\Lambda_{1} \times \ldots \times \Lambda_{n}$ and pmp actions $\Lambda_{i} \curvearrowright Y_{i}$, positive numbers $t_{i}>0$ with $t_{1} \ldots t_{n}=t /\left[\Lambda: \Lambda_{0}\right]$ and a unitary $u \in M^{t /\left[\Lambda: \Lambda_{0}\right]}$ such that $\Lambda_{0} \curvearrowright Y_{0}$ is isomorphic to the product action $\Lambda_{1} \times \ldots \times \Lambda_{n} \curvearrowright Y_{1} \times \ldots \times Y_{n}$ and $u\left(L^{\infty}\left(Y_{i}\right) \rtimes \Lambda_{i}\right) u^{*}=M_{i}^{t_{i}}$, for every $1 \leq i \leq n$.

An essential ingredient of the proof of Theorem 4.1 consists of applying A. Ioana's ultrapower technique [Io11, which we recall in the following form. This result is essentially contained in the proof of [Io11, Theorem 3.1] and its statement is roughly [DHI16, Theorem 4.1]. We leave the proof to the reader, since it follows verbatim the proof of the result in DHI16.

Theorem 4.2 ([Io11]). Let $M=B \rtimes \Lambda$ be a II $I_{1}$ factor, where $\Lambda \curvearrowright B$ is a trace preserving action on a tracial von Neumann algebra. Let $\Delta: M \rightarrow M \bar{\otimes} M$ be the $*$-homomorphism defined by $\Delta(b)=b \otimes 1$ and $\Delta\left(b v_{\lambda}\right)=b v_{\lambda} \otimes v_{\lambda}$, for all $b \in B$ and $\lambda \in \Lambda$. Let $P, Q \subset M$ be von Neumann subalgebras such that $\Delta(P) \prec_{M \bar{\otimes} M} M \bar{\otimes} Q$.

Then there exists a decreasing sequence of subgroups $\Sigma_{k}<\Lambda$ such that $P \prec_{M} B \rtimes \Sigma_{k}$, for every $k \geq 1$, and $Q^{\prime} \cap M \prec_{M} B \rtimes\left(\cup_{k \geq 1} C_{\Lambda}\left(\Sigma_{k}\right)\right)$.

Note that the ultrapower technique has recently been used in several other works [CdSS15, KV15, DHI16, CI17, CU18.

Proof of Theorem 4.1. We start the proof by fixing some notation. Let $l \geq t$ be an integer and let $p \in L^{\infty}(X \times \mathbb{Z} / l \mathbb{Z})$ be a projection of trace $t / l$ such that $M^{t}=p\left(L^{\infty}(X \times \mathbb{Z} / l \mathbb{Z}) \rtimes(\Gamma \times \mathbb{Z} / l \mathbb{Z})\right) p$. For ease of notation, we assume that $0<t \leq 1$, and therefore we can take $l=1$. Hence, $p M p=$ $L^{\infty}(Y) \rtimes \Lambda$.

For any $1 \leq i \leq n$, let $\hat{i}=\{1, \ldots, n\} \backslash\{i\}$ and $A_{i}=L^{\infty}\left(X_{i}\right)$. For a subset $F \subset\{1, \ldots, n\}$, we denote $\Gamma_{F}=\times_{i \in F} \Gamma_{i}, A_{F}=\bar{\otimes}_{i \in F} A_{i}$ and $M_{F}=\bar{\otimes}_{i \in F} M_{i}$. Since the groups $\Gamma_{i}$ 's are weakly amenable 
and biexact, PV12, Theorem 1.3] implies that $M$ has a unique Cartan subalgebra up to unitarily conjugacy. Hence, we may assume that $L^{\infty}(Y)=L^{\infty}(X) p$. Denote $A=L^{\infty}(X)$ and $B=L^{\infty}(Y)$. Following [PV09] we define the comultiplication $\Delta: M^{t} \rightarrow M^{t} \bar{\otimes} L(\Lambda)$ by $\Delta\left(b v_{\lambda}\right)=b v_{\lambda} \otimes v_{\lambda}$, for all $b \in L^{\infty}(Y)$ and $\lambda \in \Lambda$. Since $M=M_{\hat{j}} \bar{\otimes} M_{j}$, we can write $M^{t}=M_{\hat{j}} \bar{\otimes} M_{j}^{t}$, for every $1 \leq j \leq n$. The proof of the theorem is divided between the following four claims.

Claim 1. We can find $1 \leq j_{0} \leq n$ such that $\Delta\left(L\left(\Gamma_{\hat{n}}\right)\right) \prec_{M^{t} \bar{\otimes} M^{t}} M^{t} \bar{\otimes} M_{\hat{j}_{0}}$.

Proof of Claim 1. First, remark that there exists $1 \leq j_{0} \leq n$ such that $\Delta\left(M_{n}^{t}\right)$ is not amenable relative to $M^{t} \bar{\otimes} M_{\hat{j}_{0}}$ inside $M^{t} \bar{\otimes} M^{t}$. Otherwise, by applying Proposition 2.15(2), we obtain that $\Delta\left(M_{n}^{t}\right)$ is amenable relative to $M^{t} \otimes 1$ inside $M^{t} \bar{\otimes} M^{t}$. This implies by [IPV10, Lemma 10.2] that $M_{n}$ is amenable, contradiction. Therefore, by using the fact that $\Gamma_{j_{0}} \in \mathcal{C}_{r s s}$, we obtain that $\Delta\left(M_{\hat{n}}\right) \prec_{M^{t} \bar{\otimes} M} M^{t} \bar{\otimes}\left(A_{j_{0}} \bar{\otimes} M_{\hat{j}_{0}}\right)$. Since $\Gamma_{\hat{n}}$ has property $(\mathrm{T})$, Lemma 2.7 shows that $\Delta\left(L\left(\Gamma_{\hat{n}}\right)\right) \prec_{M^{t} \bar{\otimes} M^{t}}$ $M^{t} \bar{\otimes} M_{\hat{j}_{0}}$.

We are now in a position to apply the ultrapower technique from [Io11. Combining Claim 1 with Theorem 4.2, we deduce the existence of a decreasing sequence of subgroups $\Sigma_{k}<\Lambda$ such that

$$
L\left(\Gamma_{\hat{n}}\right) \prec_{M^{t}} B \rtimes \Sigma_{k} \text {, for every } k \geq 1 \text {, and } M_{j_{0}}^{t} \prec_{M^{t}} B \rtimes\left(\cup_{k \geq 1} C_{\Lambda}\left(\Sigma_{k}\right)\right) \text {. }
$$

Claim 2. There exists a non-amenable subgroup $\Sigma<\Lambda$ with non-amenable centralizer $C_{\Lambda}(\Sigma)$ and a projection $e \in \mathcal{N}_{p M p}(B \rtimes \Sigma)^{\prime} \cap p M p$ such that

$$
A \rtimes \Gamma_{\hat{n}} \prec_{M}^{s^{\prime}}(B \rtimes \Sigma) e \text { and }(B \rtimes \Sigma) e \prec_{M}^{s} A \rtimes \Gamma_{\hat{n}} .
$$

Proof of Claim 2. Relation (4.1) implies that there exists a non-amenable subgroup $\Sigma<\Lambda$ with non-amenable centralizer $C_{\Lambda}(\Sigma)$ such that $L\left(\Gamma_{\hat{n}}\right) \prec_{M} B \rtimes \Sigma$. We can use Lemma 2.14(1) and derive that $A \rtimes \Gamma_{\hat{n}} \prec_{M} B \rtimes \Sigma$. By applying Lemma 2.5)(2), there exists a non-zero projection $e \in \mathcal{N}_{p M p}(B \rtimes \Sigma)^{\prime} \cap p M p$ such that $A \rtimes \Gamma_{\hat{n}} \prec_{M}^{s^{\prime}}(B \rtimes \Sigma) e$.

For proving the claim, it remains to show that $(B \rtimes \Sigma) e \prec_{M}^{s} A \rtimes \Gamma_{\hat{n}}$. To this end, take a projection $f \in \mathcal{N}_{p M p}(B \rtimes \Sigma)^{\prime} \cap p M p \subset B^{\Sigma C_{\Lambda}(\Sigma)}$ with $f \leq e$. First, note that $L\left(C_{\Lambda}(\Sigma)\right) f$ is amenable relative to $A \rtimes \Gamma_{n}$. Indeed, take an arbitrary $1 \leq i \leq n-1$. Since $\Gamma_{i}$ belongs to $\mathcal{C}_{r s s}$, Lemma 2.16 implies that $L(\Sigma) f \prec_{M} A \rtimes \Gamma_{\hat{i}}$ or $L\left(C_{\Lambda}(\Sigma)\right) f$ is amenable relative to $A \rtimes \Gamma_{\hat{i}}$. If the former relation holds, we can apply Lemma 2.14(1) and obtain that $(B \rtimes \Sigma) f \prec_{M} A \rtimes \Gamma_{\hat{i}}$. In combination with $A \rtimes \Gamma_{\hat{n}} \prec_{M}^{s^{\prime}}(B \rtimes \Sigma) f$, Lemma 2.4(2) implies that $A \rtimes \Gamma_{\hat{n}} \prec_{M} A \rtimes \Gamma_{\hat{i}}$, contradicting the fact that $\Gamma_{i}$ is an infinite group. Therefore, $L\left(C_{\Lambda}(\Sigma)\right) f$ is amenable relative to $A \rtimes \Gamma_{\hat{i}}$, for all $1 \leq i \leq n-1$. By applying Proposition 2.15(2), we obtain that $L\left(C_{\Lambda}(\Sigma)\right) f$ is amenable relative to $A \rtimes \Gamma_{n}$.

Since $\Gamma_{n}$ belongs to $\mathcal{C}_{r s s}$, we apply once again Lemma 2.16 and obtain that $L\left(C_{\Lambda}(\Sigma)\right) f$ is amenable relative to $A \rtimes \Gamma_{\hat{n}}$ or $L(\Sigma) f \prec_{M} A \rtimes \Gamma_{\hat{n}}$. The former relation combined with the fact that $L\left(C_{\Lambda}(\Sigma)\right) f$ is amenable relative to $A \rtimes \Gamma_{n}$ gives that $C_{\Lambda}(\Sigma)$ is amenable by Proposition 2.15)(2), contradiction. Hence, $L(\Sigma) f \prec_{M} A \rtimes \Gamma_{\hat{n}}$. By applying Lemma 2.14(1), we obtain that $(B \rtimes \Sigma) f \prec_{M} A \rtimes \Gamma_{\hat{n}}$. This shows that $(B \rtimes \Sigma) e \prec_{M}^{s} A \rtimes \Gamma_{\hat{n}}$, which ends the proof of the claim.

Remark that Claim 2 allows us to apply Proposition 2.9 and derive that $\Sigma$ is measure equivalent to $\Gamma_{\hat{n}}$. Since property $(\mathrm{T})$ is a measure equivalence invariant [Fu99a, Corollary 1.4], we deduce that $\Sigma$ has property $(\mathrm{T})$ as well.

Denote $\Delta=\left\{g \in \Lambda \mid \mathcal{O}_{\Sigma}(g)\right.$ is finite $\}$. Note that $\Delta$ is normalized by $\Sigma$ and $L(\Sigma)^{\prime} \cap(B \rtimes \Lambda) \subset B \rtimes \Delta$.

Claim 3. $B^{\Delta \Sigma} e$ is completely atomic and $\Delta \Sigma$ is a finite index subgroup of $\Lambda$.

Proof of Claim 3. First, we show that $B^{\Delta \Sigma} e$ is completely atomic and use this to derive the second part of the claim. Claim 2 implies that $A \rtimes \Gamma_{\hat{n}} \prec_{M}(B \rtimes \Delta \Sigma) f$, for all $f \in B^{\Delta \Sigma}$ such that $f \leq e$. 
By passing to relative commutants and by applying Lemma 2.5 (3), we obtain that $B^{\Delta \Sigma} f \prec_{M} A_{n}$. Notice that $\mathcal{N}_{e M e}\left(B^{\Delta \Sigma} e\right)^{\prime} \cap e M e \subset B^{\Delta \Sigma} e$, and hence, Lemma 2.5)(1) gives that $B^{\Delta \Sigma} e \prec_{M}^{s} A_{n}$.

Now we show that $B^{\Delta \Sigma} e \prec_{M}^{s} M_{\hat{n}}$. Take a non-zero projection $f \in B^{\Delta \Sigma}$ with $f \leq e$. Claim 2 implies that $L(\Sigma) f \prec_{M} A \rtimes \Gamma_{\hat{n}}$. Since $\Sigma$ has property (T), by using Lemma 2.7 we deduce that $L(\Sigma) f \prec_{M} M_{\hat{n}}$. By passing to relative commutants and by applying Lemma 2.5)(3), it follows that $\left(\left(L(\Sigma)^{\prime} \cap p M p\right)^{\prime} \cap p M p\right) f \prec_{M} M_{\hat{n}}$. Since $L(\Sigma)^{\prime} \cap p M p \subset B \rtimes \Delta$, we get $B^{\Sigma \Delta} f \prec_{M} M_{\hat{n}}$. Therefore, $B^{\Delta \Sigma} e \prec_{M}^{s} M_{\hat{n}}$. Together with the conclusion of the previous paragraph, Proposition 2.15)(1) shows that $B^{\Delta \Sigma} e \prec_{M}^{s} \mathbb{C} 1$, which implies that $B^{\Delta \Sigma} e$ is completely atomic.

We continue by taking a non-zero projection $e_{0} \in B^{\Delta \Sigma}$ with $e_{0} \leq e$ such that $B^{\Delta \Sigma} e_{0}=\mathbb{C} e_{0}$. On one hand, by combining Lemma 2.7 and Claim 2 it follows that $L(\Sigma) e_{0} \prec_{M} M_{\hat{n}}$. By considering relative commutants Lemma 2.5(3) implies that $M_{n} \prec_{M}(B \rtimes \Delta \Sigma) e_{0}$. On the other hand, Claim 2 gives that $M_{\hat{n}} \prec_{M}(B \rtimes \Delta \Sigma) e_{0}$. Note that $\mathcal{N}_{e_{0} M e_{0}}\left((B \rtimes \Delta \Sigma) e_{0}\right)^{\prime} \cap e_{0} M e_{0} \subset B^{\Delta \Sigma} e_{0}=\mathbb{C} e_{0}$. Therefore, by applying Lemma 2.6, we obtain that $M \prec_{M}(B \rtimes \Delta \Sigma) e_{0}$. It is easy to see that this implies $L(\Lambda) \prec_{L(\Lambda)} L(\Delta \Sigma)$. Hence, we obtain that $\Delta \Sigma$ is a finite index subgroup of $\Lambda$ by [DHI16, Lemma $2.5(1)]$.

Claim 4. There exists a finite index subgroup $\Sigma_{0}$ of $\Sigma$ such that $\left[\Lambda: \Sigma_{0} C_{\Lambda}\left(\Sigma_{0}\right)\right]<\infty$. Moreover, there exists a non-zero projection $q \in B^{\Sigma_{0} C_{\Lambda}\left(\Sigma_{0}\right)}$ satisfying

$$
L\left(\Sigma_{0}\right) q \prec_{M^{t}} M_{\hat{n}} \text { and } L\left(C_{\Lambda}\left(\Sigma_{0}\right)\right) q \prec_{M^{t}}^{s} M_{n}^{t} .
$$

Proof of Claim 4. Recall that $\Delta=\left\{g \in \Lambda \mid \mathcal{O}_{\Sigma}(g)\right.$ is finite $\}$. Let $\left\{\mathcal{O}_{k}\right\}_{k \in \mathbb{N}}$ be a countable enumeration of all the finite orbits of the action by conjugation of $\Sigma$ on $\Lambda$ and notice that $\Delta=\cup_{k \in \mathbb{N}} \mathcal{O}_{k}$. Denote $\mathcal{S}_{k}=\cup_{i=1}^{k} \mathcal{O}_{i}$ and note that $\Delta_{k}:=\left\langle\mathcal{S}_{k}\right\rangle$ is an ascending sequence of subgroups of $\Lambda$ normalized by $\Sigma$ satisfying $\cup_{k} \Delta_{k}=\Delta$.

Since $\Lambda$ is measure equivalent to a property (T) group and $[\Lambda: \Delta \Sigma]<\infty$, we derive by [Fu99a, Corollary 1.4] that $\Delta \Sigma$ has property (T) as well. Therefore, there exists $k \in \mathbb{N}$ such that $\Delta \Sigma=\Delta_{k} \Sigma$. Since $\mathcal{S}_{k}$ is a finite set, then the subgroup $\Sigma_{0}:=\cap_{g \in \mathcal{S}_{k}} C_{\Sigma}(g)$ has finite index in $\Sigma$ and commutes with $\Delta_{k}$. Thus, $\left[\Lambda: \Delta_{k} \Sigma_{0}\right]<\infty$, which shows that $\left[\Lambda: \Sigma_{0} C_{\Lambda}\left(\Sigma_{0}\right)\right]<\infty$.

For proving the moreover part, remark first that as in Claim 2 we can find a non-zero projection $q \in \mathcal{N}_{p M p}\left(B \rtimes \Sigma_{0}\right)^{\prime} \cap p M p \subset B^{\Sigma_{0} C_{\Lambda}\left(\Sigma_{0}\right)}$ such that

$$
A \rtimes \Gamma_{\hat{n}} \prec_{M}^{s^{\prime}}\left(B \rtimes \Sigma_{0}\right) q \text { and }\left(B \rtimes \Sigma_{0}\right) q \prec_{M}^{s} A \rtimes \Gamma_{\hat{n}} .
$$

We continue by showing that $\left(B \rtimes C_{\Lambda}\left(\Sigma_{0}\right)\right) q \prec_{M}^{s} A \rtimes \Gamma_{n}$. Let $f \in \mathcal{N}_{p M p}\left(B \rtimes C_{\Lambda}\left(\Sigma_{0}\right)\right)^{\prime} \cap p M p \subset$ $B^{\Sigma_{0} C_{\Lambda}\left(\Sigma_{0}\right)}$ such that $f \leq q$. First, note that $L\left(\Sigma_{0}\right) f \nprec_{M} A \rtimes \Gamma_{\hat{i}}$, for any $1 \leq i \leq n-1$. Indeed, if there exists such an $i$ for which $L\left(\Sigma_{0}\right) f \prec_{M} A \rtimes \Gamma_{\hat{i}}$, we get by Lemma 2.14(1) that $\left(B \rtimes \Sigma_{0}\right) f \prec_{M} A \rtimes \Gamma_{\hat{i}}$. By using Lemma 2.4(2), relation (4.2) shows that $\Gamma_{i}$ is a finite group, contradiction.

Note that actually $L\left(\Sigma_{0}\right) f$ is not amenable relative to $A \rtimes \Gamma_{\hat{i}}$, for any $1 \leq i \leq n-1$. Indeed, if there exists such an $i$, by using that $\Gamma_{i} \in \mathcal{C}_{r s s}$, we get that $L\left(\Sigma_{0} C_{\Lambda}\left(\Sigma_{0}\right)\right) f$ is amenable relative to $A \rtimes \Gamma_{\hat{i}}$ since $L\left(\Sigma_{0}\right) f \nprec_{M} A \rtimes \Gamma_{\hat{i}}$. By using Lemma 2.14(2) we obtain that $B \rtimes\left(\Sigma_{0} C_{\Lambda}\left(\Sigma_{0}\right)\right) f_{0}$ is amenable relative to $A \rtimes \Gamma_{\hat{i}}$, for some non-zero projection $f_{0} \in B^{\Sigma_{0} C_{\Lambda}\left(\Sigma_{0}\right)}$. By combining Lemma 2.12. Lemma 2.10 and [DHI16, Lemma 2.6(3)], it follows that $f_{0}(B \rtimes \Lambda) f_{0}$ is amenable relative to $B \rtimes\left(\Sigma_{0} C_{\Lambda}\left(\Sigma_{0}\right)\right) f_{0}$ inside $M$. By applying [OP07, Proposition 2.4(3)] and [DHI16, Lemma 2.6(2)] we get that $A \rtimes \Gamma$ is amenable relative to $A \rtimes \Gamma_{\hat{i}}$. Hence, OP07, Proposition 2.4(1)] shows that $\Gamma_{i}$ is amenable, false.

Now, we are finally showing that $\left(B \rtimes C_{\Lambda}\left(\Sigma_{0}\right)\right) f \prec_{M}^{s} A \rtimes \Gamma_{n}$. Let $1 \leq i \leq n-1$. By using once again that $\Gamma_{i} \in \mathcal{C}_{r s s}$ we deduce by Lemma 2.16 that $L\left(C_{\Lambda}\left(\Sigma_{0}\right)\right) f \prec_{M} A \rtimes \Gamma_{\hat{i}}$ since $L\left(\Sigma_{0}\right) f$ is not amenable relative to $A \rtimes \Gamma_{\hat{i}}$ inside $M$. Note that $\left(B \rtimes C_{\Lambda}\left(\Sigma_{0}\right)\right) f \prec_{M} A \rtimes \Gamma_{\hat{i}}$ by Lemma 2.14](1), 
and hence $\left(B \rtimes C_{\Lambda}\left(\Sigma_{0}\right)\right) q \prec_{M}^{s} A \rtimes \Gamma_{\hat{i}}$ by Lemma 2.5(1). We can apply now Proposition 2.15(1) and deduce that $\left(B \rtimes C_{\Lambda}\left(\Sigma_{0}\right)\right) q \prec_{M}^{s} A \rtimes \Gamma_{n}$.

By applying Lemma 2.4(1), we derive that $L\left(\Sigma_{0}\right) q \prec_{M}^{s} A \rtimes \Gamma_{\hat{n}}$ and $L\left(C_{\Lambda}\left(\Sigma_{0}\right)\right) q \prec_{M}^{s} A \rtimes \Gamma_{n}$. Since the groups $\Sigma_{0}$ and $C_{\Lambda}\left(\Sigma_{0}\right)$ have property (T), we can use Lemma 2.7 and obtain the claim.

Finally, by applying Theorem 3.1 we obtain that there exist commuting subgroups $\Lambda_{1}^{n-1}, \Lambda_{n}<\Lambda$ such that $\Lambda \curvearrowright Y$ is induced from an action $\Lambda_{1}^{n-1} \Lambda_{n} \curvearrowright Y_{n}^{n}$. Denote $p_{n}=1_{Y_{n}^{n}} \in B^{\Lambda_{1}^{n-1} \Lambda_{n}}$. Moreover, there exist a decomposition $M^{\tau\left(p_{n}\right)}=M_{\hat{n}}^{t_{1}^{n-1}} \bar{\otimes} M_{n}^{t_{n}}$ for some $t_{1}^{n-1}, t_{n}>0$ with $t_{1}^{n-1} t_{n}=\tau\left(p_{n}\right)$, and a unitary $u_{n} \in M^{\tau\left(p_{n}\right)}$ such that:

$$
u_{n}\left(B^{\Lambda_{n}} \rtimes \Lambda_{1}^{n-1}\right) p_{n} u_{n}^{*}=M_{\hat{n}}^{t_{1}^{n-1}} \text { and } u_{n}\left(B^{\Lambda_{1}^{n-1}} \rtimes \Lambda_{n}\right) p_{n} u_{n}^{*}=M_{n}^{t_{n}} .
$$

In particular, there exist pmp actions $\Lambda_{1}^{n-1} \Lambda_{n} \curvearrowright Y_{1}^{n-1}$ and $\Lambda_{n} \curvearrowright Y_{n}$ such that

$$
u_{n}\left(L^{\infty}\left(Y_{1}^{n-1}\right) \rtimes \Lambda_{1}^{n-1}\right) u_{n}^{*}=M_{\hat{n}}^{t_{1}^{n-1}} \text { and } u_{n}\left(L^{\infty}\left(Y_{n}\right) \rtimes \Lambda_{n}\right) u_{n}^{*}=M_{n}^{t_{n}},
$$

and $\Lambda_{1}^{n-1} \Lambda_{n} \curvearrowright Y_{n}^{n}$ is isomorphic to the product action $\Lambda_{1}^{n-1} \times \Lambda_{n} \curvearrowright Y_{1}^{n-1} \times Y_{n}$.

Applying an induction argument and Remark 1.2 to relation (4.3), it is easy to see that the conclusion follows.

Before we proceed with the proof of Theorem $\mathrm{A}$, we recall some notation. Assume that $M$ is a $\mathrm{II}_{1}$ factor and $A \subset M$ is a Cartan subalgebra, i.e. a maximal abelian regular von Neumann subalgebra. The inclusion $A^{t} \subset M^{t}$ is defined as the isomorphism class of the inclusion $p\left(\ell^{\infty}(\mathbb{Z}) \bar{\otimes} A\right) p \subset p\left(\mathbb{B}\left(\ell^{2}(\mathbb{Z})\right) \bar{\otimes} M\right) p$, where $p \in \mathbb{B}\left(\ell^{2}(\mathbb{Z})\right) \bar{\otimes} M$ is a projection satisfying $(\operatorname{Tr} \otimes \tau)(p)=t$. Here, we denote by $\ell^{\infty}(\mathbb{Z}) \subset \mathbb{B}\left(\ell^{2}(\mathbb{Z})\right)$ the subalgebra of diagonal operators and by $\operatorname{Tr}$ the usual trace on $\mathbb{B}\left(\ell^{2}(\mathbb{Z})\right)$.

Proof of Theorem A. For any $1 \leq i \leq n$, denote $M_{i}=L^{\infty}\left(X_{i}\right) \rtimes \Gamma_{i}$. Applying Theorem 4.1, we obtain that that $\Lambda \curvearrowright Y$ is induced from an action $\Lambda_{0} \curvearrowright Y_{0}$ and there exist a decomposition $\Lambda_{0}=\Lambda_{1} \times \ldots \times \Lambda_{n}$ and pmp actions $\Lambda_{i} \curvearrowright Y_{i}$, positive numbers $t_{i}>0$ with $t_{1} \ldots t_{n}=t /\left[\Lambda: \Lambda_{0}\right]$ and a unitary $u \in M^{t /\left[\Lambda: \Lambda_{0}\right]}$ such that $\Lambda_{0} \curvearrowright Y_{0}$ is isomorphic to $\Lambda_{1} \times \ldots \times \Lambda_{n} \curvearrowright Y_{1} \times \ldots \times Y_{n}$ and $u\left(L^{\infty}\left(Y_{i}\right) \rtimes \Lambda_{i}\right) u^{*}=M_{i}^{t_{i}}$, for all $1 \leq i \leq n$. Note that $L^{\infty}\left(Y_{i}\right) \prec_{M} L^{\infty}(X)$ and $u L^{\infty}\left(Y_{i}\right) u^{*} \subset M_{i}^{t_{i}}$, for any $1 \leq i \leq n$. This implies that $u L^{\infty}\left(Y_{i}\right) u^{*} \prec_{M_{i}^{t_{i}}} L^{\infty}\left(X_{i}\right)^{t_{i}}$. Remark that $u L^{\infty}\left(Y_{i}\right) u^{*}$ and $L^{\infty}\left(X_{i}\right)^{t_{i}}$ are Cartan subalgebras of $M_{i}^{t_{i}}$. Hence, by applying [Po01, Theorem A.1] and [Po01, Proposition in p.829], we get that $u L^{\infty}\left(Y_{i}\right) u^{*}$ is unitarily conjugate to $L^{\infty}\left(X_{i}\right)^{t_{i}}$ inside $M_{i}^{t_{i}}$. Using [FM75, it follows that $\Lambda_{i} \curvearrowright Y_{i}$ is stably orbit equivalent to $\Gamma_{i} \curvearrowright X_{i}$ with index $t_{i}$.

\section{REFERENCES}

[Be14] M. Berbec: $W^{*}$-superrigidity for wreath products with groups having positive first $\ell^{2}$-Betti number, Internat. J. Math 26 (2015), no. 1, 1550003, 27pp.

[BV12] M. Berbec, S. Vaes: $W^{*}$-superrigidity for group von Neumann algebras of left-right wreath products, Proceedings of the London Mathematical Society (3) 108 (2014), no. 5, 1116-1152.

[CdSS15] I. Chifan, R. de Santiago, T. Sinclair: $W^{*}$-rigidity for the von Neumann algebras of products of hyperbolic groups, Geom. Funct. Anal. 26 (2016), no. 1, 136-159.

[CdSS17] I. Chifan, R. de Santiago, W. Sucpikarnon: Tensor product decompositions of II $I_{1}$ factors arising from extensions of amalgamated free product groups, Communications in Mathematical Physics 364 (2018), 11631194.

[CFW81] A. Connes, J. Feldman, B. Weiss: An amenable equivalence relations is generated by a single transformation, Ergodic Th. Dynam. Sys. 1(1981), 431-450.

[CI08] I. Chifan, A. Ioana: Ergodic subequivalence relations induced by a Bernoulli action, Geom. Funct. Anal. 20 (2010), no. 1, 53-67. 
[CI17] I. Chifan, A. Ioana: Amalgamated Free Product Rigidity for Group von Neumann Algebras. Adv. Math. 329 (2018).

[CIK13] I. Chifan, Y. Kida, A. Ioana: $W^{*}$-superrigidity for arbitrary actions of central quotients of braid groups, Math. Ann. 361 (2015), no. 3-4, 563-582.

[CK15] I. Chifan, Y. Kida: $O E$ and $W^{*}$ superrigidity results for actions by surface braid groups, Proceedings of the London Mathematical Society, in press.

[CJ85] A. Connes, V. F. R. Jones, Property (T) for von Neumann algebras, Bull. Lond. Math. Soc. 17 (1985), $57-62$.

[CU18] I. Chifan, B. Udrea: Some rigidity results for $I I_{1}$ factors arising from wreath products of property (T) groups, preprint arXiv:1804.04558.

[DHI16] D. Drimbe, D. Hoff, A. Ioana: Prime II factors arising from irreducible lattices in product of rank one simple Lie groups, to appear in J. Reine. Angew. Math, arXiv: 1611.02209 (2016).

[Dr15] D. Drimbe: Cocycle and orbit equivalence superrigidity for coinduced actions, Ergodic Theory and Dynamical Systems 38 (2018), no. 7, 2644-2665.

[Dr19] D. Drimbe: Prime II factors arising from actions of product groups, preprint arXiv 1904.06637.

[Dy58] H. Dye: On groups of measure preserving transformation. I, Amer. J. Math. 81 (1959), 119-159.

[FM75] J. Feldman, C. Moore: Ergodic equivalence relations, cohomology, and von Neumann algebras. II, Trans. Amer. Math. Soc. 234 (1977), no. 2, 325-359. MR 0578730 (58 \#28261b)

[Fu99a] A. Furman: Gromov's measure equivalence and rigidity of higher rank lattices, Ann. of Math. 150 (1999), 1059-1081.

[Fu99b] A. Furman: Orbit equivalence rigidity, Ann. of Math. (2) 150 (1999), no. 3, 1083-1108.

[Fu09] A. Furman: A survey of Measured Group Theory, Geometry, Rigidity, and Group Actions, 296-374, The University of Chicago Press, Chicago and London, 2011.

[Ga99] D. Gaboriau: Coût des relations d'équivalence et de groupes. Invet. Math. 139 (2000), no. 41-98.

[Ga10] D. Gaboriau: Orbit equivalence and measured group theory, In Proceedings of the ICM (Hyderabad, India, 2010), Vol. III. Hindustan Book Agency, 2010, pp. 1501-1527.

[Ge95] L. Ge: On maximal injective subalgebras of factors, Adv. Math. 118 (1996), no. 1, 34-70.

[GITD16] D. Gaboriau, A. Ioana, R. Tucker-Drob: Cocycle superrigidity for translation actions of product groups, to appear in the American Journal of Mathematics.

[Gr91] M. Gromov: Asymptotic invariants of infinite groups, Geometric group theory, Vol. 2 (Sussex, 1991), London Math. Soc. Lecture Note Ser., vol. 182, Cambridge Univ. Press, Cambridge, 1993, pp. 1-295.

[Gr93] M. Gromov: Asymptotic invariants of infinite groups. In Geometric group theory, Vol. 2 (Sussex, 1991), pages 1295. Cambridge Univ. Press, Cambridge, 1993. MR1253544 (95m:20041).

[HPV11] C. Houdayer, S. Popa, S. Vaes: A class of groups for which every action is $W^{*}$-superrigid, Groups Geom. Dyn. 7 (2013), 577-590.

[Io08] A. Ioana: Cocycle superrigidity for profinite actions of property (T) groups, Duke Math J. 157, (2011), 337-367.

[Io11] A. Ioana: Uniqueness of the group measure space decomposition for Popa's $\mathcal{H} \mathcal{T}$ factors, Geom. Funct. Anal. 22 (2012), no. 3, 699-732.

[Io12] A. Ioana: Classification and rigidity for von Neumann algebras, European Congress of Mathematics, EMS (2013), 601-625.

[Io14] A. Ioana: Strong ergodicity, property (T), and orbit equivalence rigidity for translation actions, J. Reine. Angew. Math.

[Io17] A. Ioana: Rigidity for von Neumann algebras, arXiv: 1712.00151.

[IPV10] A. Ioana, S. Popa, S. Vaes: A class of superrigid group von Neumann algebras, Ann. of Math. (2) 178 (2013), no. 1, 231-286.

[Jo81] V.F.R. Jones, Index for subfactors, Invent. Math. 72 (1983), 125.

[Ki06] Y. Kida: Measure equivalence rigidity of the mapping class group, Ann. of Math. (2) 171 (2010), 1851-1901.

[Ki09] Y. Kida: Rigidity of amalgamated free products in measure equivalence, J. Topol. 4 (2011), $687-735$.

[KV15] A. Krogager, S. Vaes: A class of $I_{1}$ factors with exactly two crossed product decompositions, preprint arXiv:1512.06677, to appear in J. Mathématiques Pures et Appliquées.

[MS02] N.Monod, Y. Shalom: Orbit equivalence rigidity and bounded cohomology, Ann. of Math. 164 (2006), 825878.

[MvN44] F.J. Murray, J. von Neumann: Rings of operators, IV. Ann. Math. 44 (1943), 716-808

[OP03] N. Ozawa, S. Popa: Some prime factorization results for type $I I_{1}$ factors, Invent. Math. 156 (2004), no. 2, 223-234. pp.

[OP07] N. Ozawa, S. Popa: On a class of $I_{1}$ factors with at most one Cartan subalgebra, Ann. of Math. (2) 172 (2010), no. 1, 713-749. 
[OW80] D. Ornstein, B. Weiss: Ergodic theory of amenable group actions. I. The Rohlin lemma, Bull. Amer. Math. Soc. (N.S.), 2(1):161-164, 1980.

[Pe06] J. Peterson: $L^{2}$-rigidity in von Neumann algebras, Invent. Math. 175 (2009), no. 2, 417-433.

[Po83] S. Popa: Orthogonal pairs of *-subalgebras in finite von Neumann algebras, J. Operator Theory 9 (1983), no. 2, 253-268.

[Po01] S. Popa: On a class of type II factors with Betti numbers invariants, Ann. of Math. 163 (2006), 809-899.

[Po03] S. Popa: Strong rigidity of $I I_{1}$ factors arising from malleable actions of w-rigid groups. I., Invent. Math. 165 (2006), no. 2, 369-408.

[Po05] S. Popa: Cocycle and orbit equivalence superrigidity for malleable actions of w-rigid groups, Invent. Math. 170 (2007), 243-295.

[Po06] S. Popa: On the superrigidity of malleable actions with spectral gap, J. Amer. Math. Soc. 21 (2008), 9811000 .

[Po07] S. Popa: Deformation and rigidity for group actions and von Neumann algebras, In Proceedings of the ICM (Madrid, 2006), Vol. I, European Mathematical Society Publishining House, 2007, 445-477.

[PP86] M. Pimsner, S. Popa: Entropy and index for subfactors, Ann. Sci. École Norm. Sup. 19 (1986), $57=106$.

[PS09] J. Peterson, T. Sinclair: On cocycle superrigidity for Gaussian actions Erg. Th. and Dyn. Sys.32 (2012), no. 1, 249-272.

[PV08] S.Popa, S. Vaes: Cocycle and orbit superrigidity for lattices in $S L(n, \mathbb{R})$ acting on homogeneous spaces, In Geometry, rigidity and group actions, eds. B. Farb and D. Fisher, The University of Chicago Press, 182 (2011), 419-541.

[PV09] S. Popa, S. Vaes: Group measure space decomposition of $I_{1}$ factors and $W^{*}$-superrigidity, Invent. Math. 182 (2010), no. 2, 371-417.

[PV11] S. Popa, S. Vaes: Unique Cartan decomposition for II $I_{1}$ factors arising from arbitrary actions of free groups, Acta Math. 212 (2014), no. 1, 141-198.

[PV12] S. Popa, S. Vaes: Unique Cartan decomposition for II factors arising from arbitrary actions of hyperbolic groups, J. Reine Angew. Math. 694 (2014), 215-239.

[Sa09] H. Sako: Measure equivalence rigidity and bi-exactness of groups, J. Funct. Anal. 257 (2009) 3167-3202.

[Sh04] Y. Shalom: Measurable group theory, European Congress of Mathematics, European Mathematical Society Publishing House, 2005, p. 391-423.

[Si55] I. M. Singer: Automorphism of finite factors, Amer. J. Math. 77 (1955), 117-133.

[TD14] R. D. Tucker-Drob: Invariant means and the structure of inner amenable groups, Preprint. arXiv:1407.7474.

[Va08] S. Vaes: Explicit computations of all finite index bimodules for a family of II factors, Ann. Sci. Éc. Norm. Supér. (4) 41 (2008), no. 5, 743-788.

[Va10] S. Vaes: Rigidity for von Neumann algebras and their invariants, Proceedings of the ICM (Hyderabad, India, 2010), Vol. III, Hindustan Book Agency (2010), 1624-1650.

[Zi84] R. Zimmer: Ergodic theory and semisimple groups, Monographs in Mathematics, 81. Birkhauser Verlag, Basel, 1984. x+209 pp.

[Zu03] A. Zuk: Property (T) and Kazhdan constants for discrete groups, Geom. Funct. Anal., 13(3):643670, 2003.

Department of Mathematics, University of Regina, 3737 Wascana Pkwy, Regina, SK S4S 0A2, Canada.

E-mail address: daniel.drimbe@uregina.ca 